\title{
Phase Diagram of Bosonic Atoms in Two-Color Superlattices
}

\author{
Robert Roth \\ Institut für Kernphysik, Technische Universität Darmstadt, 64289 Darmstadt, Germany \\ Keith Burnett \\ Clarendon Laboratory, University of Oxford, Parks Road, Oxford OX1 3PU, United Kingdom
}

(Dated: June 16, 2018)

\begin{abstract}
We investigate the zero temperature phase diagram of a gas of bosonic atoms in one- and twocolor standing-wave lattices in the framework of the Bose-Hubbard model. We first introduce some relevant physical quantities; superfluid fraction, condensate fraction, quasimomentum distribution, and matter-wave interference pattern. We then discuss the relationships between them on the formal level and show that the superfluid fraction, which is the relevant order parameter for the superfluid to Mott-insulator transition, cannot be probed directly via the matter wave interference patterns. The formal considerations are supported by exact numerical solutions of the Bose-Hubbard model for uniform one-dimensional systems. We then map out the phase diagram of bosons in non-uniform lattices. The emphasis is on optical two-color superlattices which exhibit a sinusoidal modulation of the well depth and can be easily realized experimentally. From the study of the superfluid fraction, the energy gap, and other quantities we identify new zero-temperature phases, including a localized and a quasi Bose-glass phase, and discuss prospects for their experimental observation.

PACS numbers: 03.75.Kk, 03.75.Lm, 05.30.Jp, 73.43.Nq
\end{abstract}

\section{INTRODUCTION}

With the first experimental observation of the superfluid to Mott-insulator transition in ultracold atomic Bose gases trapped in optical lattice potentials [1, 2] the unique prospects of this new class of systems became evident. Compared to experiments with Bose-Einstein condensates in shallow magnetic traps, the use of optical standing-wave lattices gives access to a new regime which is dominated by strong correlations. The most striking ramification of these correlations are quantum phase transitions [3] which are driven by the interplay of the different contributions to the Hamiltonian of the system.

The superfluid to Mott-insulator transition is governed by competition between kinetic energy and repulsive atom-atom interaction: in the presence of strong repulsive interactions the ground state is almost a pure Fock state with a definite occupation number at the individual lattice sites. This is associated with a vanishing of the superfluid density which is the natural order parameter for superfluid to insulator phase transitions [4, 5].

New quantum phase transitions can be expected if other terms in the Hamiltonian come into play. One interesting case is the presence of some additional spatial structure of the lattice potential. This might range from a regular modulation of the well depths to random disorder. From several studies in the context of solid-state systems it is known that random disorder gives rise to new zero-temperature phases such as the Anderson localized phase and the Bose-glass phase [6, 7].

Cold atoms in optical lattices offer unparalleled possibilities to study these disorder-related phenomena [8]. By a superposition of multiple laser beams it is possible to generate a huge variety of different lattice topologies in a perfectly controlled manner. At the same time Feshbach resonances can be utilized to modify the strengths of the interatomic interaction. These powerful methods of tuning the relevant parameters are supplemented by versatile techniques to probe the state of the many-boson system, e.g. by observing the matter-wave interference pattern after the atoms were released from the lattice [1, 9] or by measuring the energy gap between the ground state and the first excited state 2]. Atoms in an optical lattice are therefore an ideal model system for the experimental investigation of the fundamental questions associated with quantum phase transitions in lattice systems.

The aim of this paper is twofold: Firstly, we want to review the important physical quantities which describe the properties of Bose gases in optical lattices and investigate the relations between them, both, analytically and numerically. The emphasis in this part of the paper is on the superfluid fraction and its connection to directly observable quantities. Secondly, we want to systematically explore the zero-temperature phase diagram for bosons in two-color superlattices.

The theoretical framework for the description of strongly correlated Bose gases in lattices is the BoseHubbard model [4], which is summarized in Sec. [I] We define the fundamental quantities, condensate fraction, quasimomentum distribution, and superfluid fraction, and discuss their relation on the formal level in Sec. III The connection of these fundamental properties to the experimental observables, e.g., the matter-wave interference pattern, is investigated in Sec. IV] The formal definition shows that the superfluid fraction depends crucially on the excitation spectrum [5]. The basic experimental observable, i.e. the interference pattern, only probes the ground state of the system and cannot therefore provide full information on the superfluid properties 
of the system. Using the example of the superfluid to Mott-insulator transition we illustrate these differences in the exact numerical solution of the Bose-Hubbard model for a one-dimensional uniform lattice. Finally, in a Sec. $\nabla$ we extend our studies to non-uniform lattice potentials. The emphasis is on so-called two-color superlattices which exhibit a sinusoidal variation of the well depths [8]. We map out the zero-temperature phase diagram as function of the interaction strength and the amplitude of the modulation and compare the behavior of number fluctuations, condensate fraction, superfluid fraction and energy gap. This enables us to identify additional zerotemperature phases, i.e. a localized phase and a quasi Bose-glass phase, and to discuss possibilities for their experimental detection.

\section{BOSE-HUBBARD MODEL}

Quantum phase transitions of Bose gases in optical lattices are associated with complex correlations in the many-body state which go far beyond a simple GrossPitaevskii-like description. A theoretical model which is capable of describing these dominating correlations is the Bose-Hubbard model. First introduced for model studies related to ${ }^{4} \mathrm{He}$ liquids in porous media and granular superconductors 10, 11] it was recently applied to ultracold atomic Bose gases in optical lattices [4].

In the following we will briefly review the assumptions which lead to the Bose-Hubbard Hamiltonian and summarize solution methods and the basic observables. Because our emphasis is on conceptual and general aspects we restrict ourselves to one-dimensional lattices, the formal generalization to two or three dimensions is straightforward.

\section{A. Bose-Hubbard Hamiltonian}

The basic assumption of the Bose-Hubbard model is that the lattice wells are sufficiently deep for the state of the system to be described using a basis of singleparticle wavefunctions, localized at the individual lattice sites. Only these localized ground states are taken into account and excited vibrational states are neglected. In the language of band structure theory the model space of the Bose-Hubbard model comprises the lowest energy band only, all excited bands are excluded. The localized single-particles states are given by the Wannier functions for the lowest band. The energy gap between the ground state band and the first excited band has therefore to be sufficiently large for admixtures from the excited bands to be negligible.

A natural way to characterize the many-boson states in the model space of the Bose-Hubbard model is the occupation number representation. Let's assume we are dealing with a system of $N$ bosons in a lattice composed of $I$ lattice sites. We introduce a set of occupation numbers $\left\{n_{1}, \ldots, n_{I}\right\}$ which specify the number of bosons in the localized single-particle state at the individual lattice sites. The set of Fock states $\left|n_{1}, \ldots, n_{I}\right\rangle$ for all possible compositions of the occupation numbers under the constraint $\sum_{i=1}^{I} n_{i}=N$ forms a complete basis the Bose-Hubbard model space.

We can define the associated creation and annihilation operators, $\hat{\mathrm{a}}_{i}^{\dagger}$ and $\hat{\mathrm{a}}_{i}$, for a boson localized at the $i$ th lattice site:

$$
\begin{aligned}
& \hat{\mathrm{a}}_{i}^{\dagger}\left|n_{1}, \ldots, n_{i}, \ldots, n_{I}\right\rangle=\sqrt{n_{i}+1}\left|n_{1}, \ldots, n_{i}+1, \ldots, n_{I}\right\rangle \\
& \hat{\mathrm{a}}_{i}\left|n_{1}, \ldots, n_{i}, \ldots, n_{I}\right\rangle=\sqrt{n_{i}}\left|n_{1}, \ldots, n_{i}-1, \ldots, n_{I}\right\rangle .
\end{aligned}
$$

The occupation number operator for the $i$ th site is given by

$$
\hat{\mathrm{n}}_{i}=\hat{\mathrm{a}}_{i}^{\dagger} \hat{\mathrm{a}}_{i}
$$

Throughout this paper the indices $i, j=1, \ldots, I$ label the lattice sites. All operator-valued quantities are marked by a hat.

Using these creation and annihilation operators one can easily translate the many-body Hamiltonian of the system - consisting of the kinetic energy, the external lattice potential, and the two-body interaction - into its second quantized form. This procedure leads directly to the Bose-Hubbard Hamiltonian for the one-dimensional lattice system

$$
\begin{aligned}
\hat{\mathrm{H}}_{0}= & -J \sum_{i=1}^{I}\left(\hat{\mathrm{a}}_{i+1}^{\dagger} \hat{\mathrm{a}}_{i}+\text { h.a. }\right) \\
& +\sum_{i=1}^{I} \epsilon_{i} \hat{\mathrm{n}}_{i}+\frac{V}{2} \sum_{i=1}^{I} \hat{\mathrm{n}}_{i}\left(\hat{\mathrm{n}}_{i}-1\right) .
\end{aligned}
$$

The first term describes the tunneling between adjacent lattice sites characterized by a strength parameter $J$. It is associated with the kinetic energy part of the first quantized Hamiltonian. In general we will use periodic boundary conditions, i.e., tunneling between the first and the last lattice site is included (the site index $I+1$ which appears in the summation is implicitly replaced by 1 ).

The second term in (3) gives an on-site single-particle energy which originates from the external potentials and the on-site part of the kinetic energy. The on-site energies $\epsilon_{i}$ are constant for a translationally invariant lattice and might be set to zero. However, in the presence of an additional parabolic trapping potential or for irregular lattices the $\epsilon_{i}$ vary with the site index $i$. Finally, the third term comprises the on-site two-body interaction characterized by the interaction strength $V$.

The parameters $J, \epsilon_{i}$, and $V$ are given by the matrix elements of the terms of the first-quantized Hamiltonian the in the localized single particle states [4, 12]. In the following we will use the tunneling parameter $J$ as the unit of energy; the dimensionless ratios $V / J$ and $\epsilon_{i} / J$ are varied to explore different regions of the zero-temperature phase diagram of the lattice system. As mentioned in 
the introduction one can envisage being able to tune the parameters of the Hamiltonian (3) experimentally, either by changing the lattice geometry or by exploiting a Feshbach resonance.

Besides the fundamental restriction of the BoseHubbard model to the ground state band some additional assumptions are used to construct the Hamiltonian (3): ( $i$ ) Only tunneling between adjacent sites is included, long range hopping over several lattice sites is neglected. (ii) Only short-range on-site interactions are included; long-range interactions as they can occur for example in the presence of dipole-dipole forces are not considered here. (iii) The tunneling strength $J$ is assumed to be independent of the site index. This is obviously the case in regular lattice potentials, but it is an approximation for irregular lattices [13]. If required all these simplifications can be easily abandoned.

\section{B. Exact solution}

The most direct way to obtain the ground state and the excitation spectrum of a zero-temperature Bose gas in one-dimensional lattices is through the exact numerical diagonalization of the Bose-Hubbard Hamiltonian. Within the occupation number basis the construction of the matrix representation of the Bose-Hubbard Hamiltonian is trivial. The only substantial hurdle is the dimension of the number basis. For fixed number of sites $I$ and number of particles $N$ the dimension of the number basis is given by

$$
D=\frac{(N+I-1) !}{N !(I-1) !} .
$$

It grows dramatically with increasing system size: For fixed filling factor $N / I=1$ the basis dimension for $I=8$ is $D=6435$, for $I=10$ it grows to $D=92378$, and for $I=12$ it reaches $D=1352078$. The Hamilton matrix is, however, extremely sparse because only the tunneling term in (3) generates off-diagonal matrix elements. Efficient iterative Lanczos-type algorithms can therefore be utilized to determine the few lowest eigenvalues and the corresponding eigenvectors. This allows to treat systems with up to $I=N=12$ on a standard PC.

As the result of the numerical solution of the eigenvalue problem we obtain the energy eigenvalues $E^{(\nu)}$ and the corresponding eigenvectors $C_{\alpha}^{(\nu)}$ for a few states $(\nu=$ $0,1,2 \ldots)$. The eigenvectors provide the coefficients of the expansion of the eigenstates in the number basis

$$
\left|\Psi_{\nu}\right\rangle=\sum_{\alpha=1}^{D} C_{\alpha}^{(\nu)}\left|\left\{n_{1}, \ldots, n_{I}\right\}_{\alpha}\right\rangle .
$$

Here the index $\alpha=1, \ldots, D$ labels the different Fock states, i.e., the different sets $\left\{n_{1}, \ldots, n_{I}\right\}_{\alpha}$ of occupation numbers.

Besides the exact numerical diagonalization, which explicitly yields the interesting state vectors, it is possible to compute selected observables using Monte-Carlo simulations [14] which allow to treat systems with several hundred lattices sites 15, 16. Because of its simplicity and transparency we will, nevertheless, restrict ourselves to the exact diagonalization for relatively small lattices, which already exhibit the important universal properties of this class of systems.

Several approximation methods have been developed to solve the Bose-Hubbard model. One of them is the so-called mean-field approximation where the state is assumed to be a direct product of independent single-site states. Thereby complex correlations between the individual sites that can be of particular importance for the description of quantum phase transition cannot be described. On top of this independent-site approximation the Gutzwiller ansatz [17] can be used to perform variational calculations of the ground state.

Another approximation scheme uses the Bogoliubov approach. Here the annihilation operators $\hat{a}_{i}$ are replaced by a complex amplitude $z_{i}$ plus a fluctuation operator $\hat{\delta}_{i}$. The fluctuation part is assumed to be small such that terms involving squares of the fluctuation operators can be neglected. The application and the limitations of this approach are discussed in [18].

\section{Simple observables}

From the ground state $\left|\Psi_{0}\right\rangle$ and a few excited states obtained from the exact diagonalization of the BoseHubbard Hamiltonian one can extract several simple observables by computing expectation values.

The simplest observable is the mean occupation number of the different sites

$$
\bar{n}_{i}=\left\langle\Psi_{0}\left|\hat{\mathrm{n}}_{i}\right| \Psi_{0}\right\rangle
$$

For a translationally invariant lattice $\bar{n}_{i}$ will be the same for all lattice sites and equal to the filling factor $N / I$ independent of the parameters $V$ and $J$. This, however, does not mean that the structure of the state does not change. A more telling quantity are the fluctuations around this mean occupation number given by

$$
\sigma_{i}=\sqrt{\left\langle\Psi_{0}\left|\hat{\mathrm{n}}_{i}^{2}\right| \Psi_{0}\right\rangle-\left\langle\Psi_{0}\left|\hat{\mathrm{n}}_{i}\right| \Psi_{0}\right\rangle^{2}}
$$

These number fluctuations provide direct information on the structure of the ground state (5). If $\left|\Psi_{0}\right\rangle$ is a superposition of many Fock states $\left|\left\{n_{1}, \ldots, n_{I}\right\}_{\alpha}\right\rangle$ then many different occupation numbers occur at the individual sites and the number fluctuations $\sigma_{i}$ will be large. If, on the other hand, the ground state is described by a single Fock state, then the fluctuations will vanish.

As a complementary measure the magnitude of the largest coefficient in the Fock state expansion (5) of the ground state can be used

$$
C_{\max }^{2}=\max \left(C_{\alpha}^{2}\right)
$$




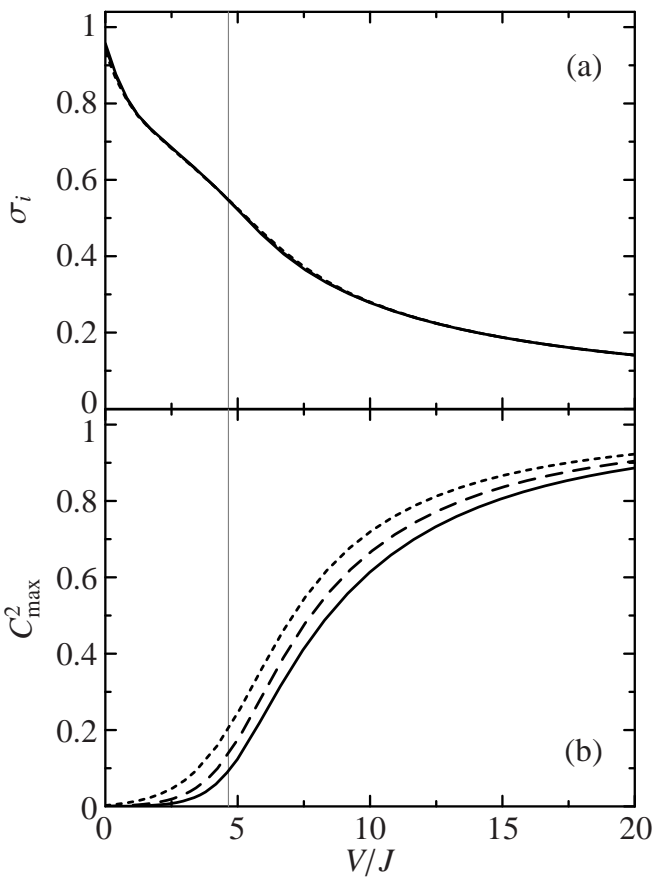

FIG. 1: Number fluctuations $\sigma_{i}$ (a) and largest coefficient $C_{\max }^{2}$ (b) for the ground state of the one-dimensional BoseHubbard model with $I=N=12$ (solid), 10 (dashed), and 8 (dotted) as function of the interaction strength $V / J$. The vertical gray line marks the critical interaction strength for the Mott-insulator transition in an infinite system [14, 19].

Although this is not directly observable it yields sensitive information on the structure of the state. A small value of $C_{\max }^{2}$ indicates that $\left|\Psi_{0}\right\rangle$ is a superposition of many number states; $C_{\max }^{2} \approx 1$ means that the ground state is a pure Fock state.

To illustrate these quantities we solve the eigenvalue problem of the Bose-Hubbard Hamiltonian (3) for a regular lattice potential $\left(\epsilon_{i}=0\right)$ with filling factor $N / I=1$ for different ratios $V / J$ of the interaction strength and the tunneling coefficient. The results for the number fluctuations $\sigma_{i}$ and the largest coefficient $C_{\max }^{2}$ are depicted in Fig. 10 The vertical gray line indicates the critical interaction strength $(V / J)_{\mathrm{cr}}=4.65$ for the superfluid to Mott-insulator transition in an infinite one-dimensional lattice extracted from a Monte Carlo calculation 14] and from a strong coupling expansion [19]. It marks the region where structural changes in the ground state are also expected in the finite size systems considered here.

Our first important observation is that the number fluctuations decrease rather slowly with increasing interaction strength. Moreover, there appears no clear signature of a phase transition in their variation. This is not an artifact of the limited lattice size, because the number fluctuation exhibit no noticeable size dependence as the three curves for $I=12,10$, and 8 in Fig. 1(a) illustrate. Thus we conclude that the number fluctuations and related quantities do not reveal the quantum phase transition from a superfluid to a Mott-insulator state and

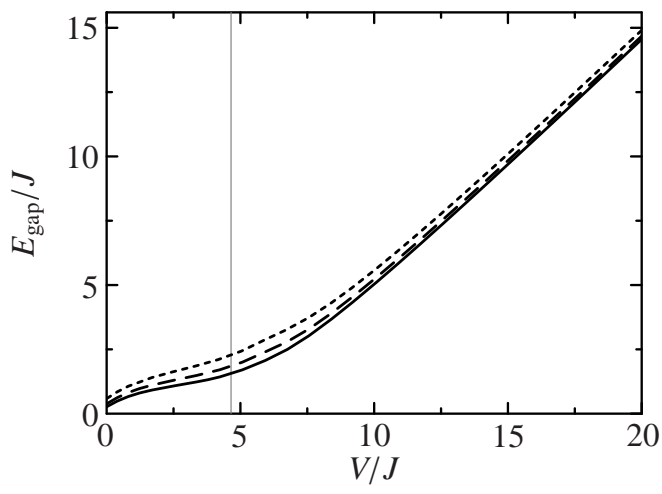

FIG. 2: Energy gap $E_{\text {gap }}$ between ground state and first excited state as function of the interaction strength $V / J$ for commensurate filling $N / I=1$ and different lattice sizes $I=12$ (solid line), 10 (dashed), 8 (dotted).

that the insulating state still has significant number fluctuations, i.e., is not a pure Fock state.

The last point is confirmed by the behavior of the largest coefficient $C_{\max }^{2}$ plotted in Fig. [1 b). This quantity is very small in the insulating phase and starts to grow in the region of the phase transition. However, even for large values of the ratio $V / J$ the largest coefficient in (15) remains smaller than 1 indicating that there is a dominant Fock state (the one with $n_{i}=N / I$ ) but that other Fock states also contribute to the ground state.

Another important observable is the energy gap, i.e., the difference between the energies of the ground state and the first excited state,

$$
E_{\text {gap }}=E^{(1)}-E^{(0)} .
$$

This quantity is of particular importance as the excited states are crucial for the superfluid properties of the system. Figure 2 depicts the behavior of the energy gap as function of the interaction strength. For small $V / J$ the energy gap is small and increases only slowly with increasing interaction strengths. The slope then starts to increase until, for $V / J \gtrsim 10$, one observes a linear increase of the energy gap with a slope 1, i.e., changing the interaction strength $V$ by a certain amount will change the energy gap by the same amount. Qualitatively, this behavior can be explained by assuming that the excited state is a superposition of different one-particle one-hole excitations of the ground state. The predominant Fock states in the expansion (5) of the excited state thus have one doubly occupied site which leads to the linear dependence of the energy gap $E_{\text {gap }}$ on $V$. These signatures of the Mott-insulator phase have been investigated experimentally [2] as well as theoretically [20, 21] by tilting the lattice potential.

The behavior of the energy gap indicates that the character of the energy spectrum changes in the region $V / J \sim 5$ to 8 . As we will see in Sec. IIIB this change is accompanied by a vanishing of superfluidity, i.e. the superfluid to Mott-insulator transition. 


\section{CONDENSATE AND SUPERFLUIDITY}

Besides the simple quantities discussed so far there are two more complex physical quantities which reveal substantial information on fundamental physical properties of the Bose gas in the lattice. These are the condensate fraction and the superfluid fraction. This section is devoted to the definition and discussion of these non-trivial quantities.

\section{A. Condensate fraction}

Unlike the Gross-Pitaevskii description of weakly interacting Bose gases [22] the exact solution of the BoseHubbard model does not assume a perfect Bose-Einstein condensate from the outset. Moreover, due to the general representation of the ground state in terms of Fock states the presence or absence of a Bose-Einstein condensate is not obvious. For example, the exact ground state (5) of a noninteracting system at zero temperature, where one expects a perfect Bose-Einstein condensate, is a superposition of many Fock states with coefficients given by the multinomial distribution.

\section{Definition}

In order to define a Bose-Einstein condensate for the ground state $\left|\Psi_{0}\right\rangle$ of a general interacting many-boson system in a lattice we consider the one-body density matrix associated with $\left|\Psi_{0}\right\rangle$

$$
\rho_{i j}^{(1)}=\left\langle\Psi_{0}\left|\hat{\mathrm{a}}_{j}^{\dagger} \hat{\mathrm{a}}_{i}\right| \Psi_{0}\right\rangle
$$

The eigenvectors of the one-body density matrix describe the so-called natural orbitals and the eigenvalues the corresponding occupation numbers.

Following the formulation of Penrose and Onsager 23] a Bose-Einstein condensate is present if one of the natural orbitals is macroscopically occupied. Its occupation number is just the number of condensate particles $N_{\mathrm{c}}$ and the eigenvector constitutes the condensate wave function $\chi_{\mathrm{c}, i}$.

Strictly speaking, macroscopic occupation of an orbital means that the ratio of its occupation number and the total particle number, in the following called condensate fraction $f_{\mathrm{c}}=N_{\mathrm{c}} / N$, remains finite in the the thermodynamic limit $(N, I \rightarrow \infty, N / I=$ const. $)$. This implies that one cannot rigorously determine the absence of a BoseEinstein condensate in finite-size systems. From the normalization of the one-body density matrix, $\operatorname{Tr} \rho_{i j}^{(1)}=N$, it follows immediately that for any system there is an eigenvalue larger or equal $N / I$. Thus the condensate fraction $f_{\mathrm{c}}$ is always larger than $1 / I$ and cannot vanish for finite-size systems. Despite of these formal complications the ratio of the largest eigenvalue of (10) and the total particle number provides important physical information

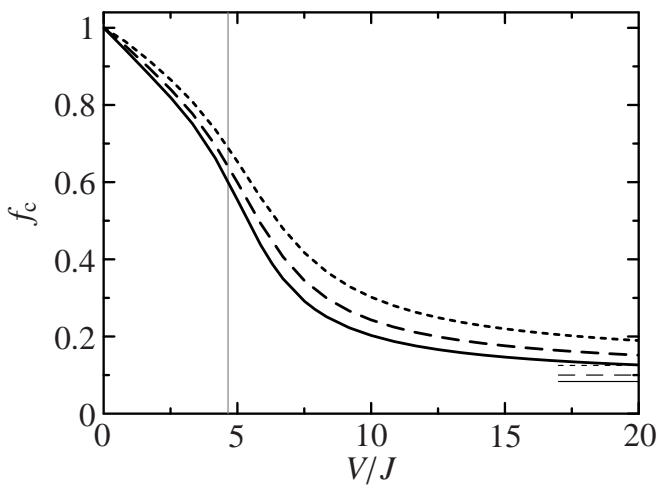

FIG. 3: Condensate fraction $f_{\mathrm{c}}$ as function of the interaction strength $V / J$ for commensurate filling $N / I=1$ and different lattice sizes $I=12$ (solid line), 10 (dashed), 8 (dotted). The thin lines at the right border indicate the asymptotic value $f_{\mathrm{c}} \rightarrow 1 / I$.

even in finite lattices. For simplicity we will use the term condensate fraction for this quantity.

The presence of a Bose-Einstein condensate implies offdiagonal long range order 24]. That is, the matrix elements of the one-body density matrix do not go to zero far off the diagonal but remain finite:

$$
\rho_{i j}^{(1)} \nrightarrow 0 \text { for }|i-j| \rightarrow \infty .
$$

The prove of this connection is straightforward if one considers the spectral decomposition of the one-body density matrix which allows us to separate the condensate part from a residual positive semi-definite density matrix $\tilde{\rho}_{i j}^{(1)}$

$$
\rho_{i j}^{(1)}=N_{\mathrm{c}} \chi_{\mathrm{c}, i}^{\star} \chi_{\mathrm{c}, j}+\tilde{\rho}_{i j}^{(1)} .
$$

It is important to realize that off-diagonal long range order is associated directly with the phenomenon of BoseEinstein condensation whereas the connection with superfluidity is, at most, indirect.

The dependence of the condensate fraction, i.e., the relative occupation of the natural orbital with largest eigenvalue, on the interaction strength $V / J$ in a regular lattice is depicted in Fig. [3] In the noninteracting case $V / J=0$ all particles are in the condensate. As soon as a finite repulsive interaction is present $(V / J>0)$ the condensate fraction is reduced, i.e., the condensate is depleted and non-condensate states are populated. For large interaction strengths the condensate fraction converges to $1 / I$. This is the reason for the pronounced size dependence of the condensate fraction in the regime of strong depletion.

\section{Quasimomentum Distribution}

The eigenvalues of the one-body density matrix (10) provide more information than just the condensate fraction. For a translationally invariant lattice one can show 
that the natural orbitals satisfy the Bloch theorem and therefore are quasimomentum eigenstates [25]. Thus the occupation numbers of the natural orbitals correspond to occupation numbers $\tilde{n}_{q}$ of the Bloch states with different quasimomenta $q$. Notice that for a finite lattice of length $L$ the quasimomenta can assume only discrete values which are integer multiples of $2 \pi / L$. The Bloch function for quasimomentum $q=0$ describes the condensate state as defined above.

An alternative way to obtain the quasimomentum distribution for a regular lattice is to use the relation between the localized Wannier functions and delocalized Bloch functions, which are the quasimomentum eigenstates. The Bloch functions $\psi_{q}(x)$ of the lowest band can be decomposed in terms of Wannier functions $w\left(x-\xi_{i}\right)$ :

$$
\psi_{q}(x)=\frac{1}{\sqrt{I}} \sum_{i=1}^{I} \mathrm{e}^{-\mathrm{i} q \xi_{i}} w\left(x-\xi_{i}\right),
$$

where $\xi_{i}$ is the coordinate of the $i$ th lattice site. Using the fact that the creation operators $\hat{a}_{i}^{\dagger}$ of the Bose-Hubbard model create a boson in the Wannier state $w\left(x-\xi_{i}\right)$ we can readily define creation operators $\hat{\mathrm{c}}_{\sigma}^{\dagger}$ for a boson in the Bloch state with quasimomentum $q[12$ ]

$$
\hat{\mathrm{c}}_{q}^{\dagger}=\frac{1}{\sqrt{I}} \sum_{i=1}^{I} \mathrm{e}^{-\mathrm{i} q \xi_{i}} \hat{\mathrm{a}}_{i}^{\dagger} .
$$

The occupation numbers of the Bloch states with quasimomentum $q$ are thus given by

$$
\begin{aligned}
\tilde{n}_{q}=\left\langle\Psi_{0}\left|\hat{\mathrm{c}}_{q}^{\dagger} \hat{\mathrm{c}}_{q}\right| \Psi_{0}\right\rangle & =\frac{1}{I} \sum_{i, j=1}^{I} \mathrm{e}^{\mathrm{i} q\left(\xi_{i}-\xi_{j}\right)}\left\langle\Psi_{0}\left|\hat{\mathrm{a}}_{j}^{\dagger} \hat{\mathrm{a}}_{i}\right| \Psi_{0}\right\rangle \\
& =\frac{1}{I} \sum_{i, j=1}^{I} \mathrm{e}^{\mathrm{i} q a(i-j)} \rho_{i j}^{(1)},
\end{aligned}
$$

where $a=\xi_{i+1}-\xi_{i}$ is the lattice spacing. As expected, the quasimomentum distribution is related to the Fourier transform of the one-body density matrix.

Figure 4 shows the occupation number for the three lowest quasimomentum states as function of the interaction strength $V / J$. The population for the $q=0$ state corresponds to the condensate fraction and is depleted as the interaction strength is increased. Simultaneously with this, the occupation numbers of Bloch states with higher quasimomenta, which vanish in the noninteracting system, increase successively. The repulsive interactions thus lead to a redistribution of the particles from the condensate to states with higher quasimomenta. In the limit of strong interactions all quasimomentum states exhibit the same occupation, i.e., the band is filled uniformly.

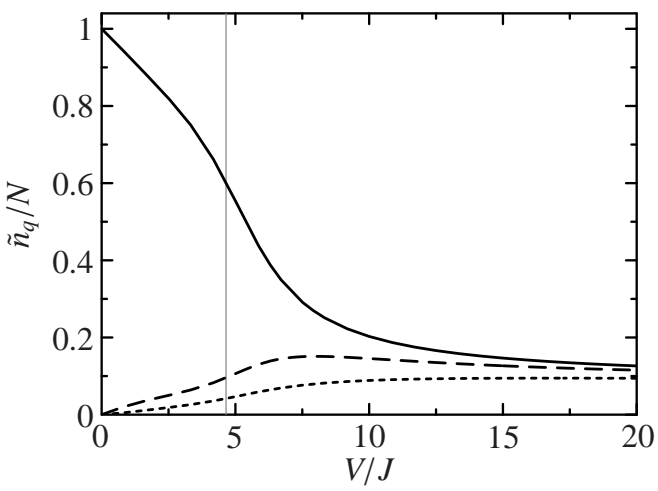

FIG. 4: Occupation numbers $\tilde{n}_{q}$ of Bloch states with quasimomentum $q$ function of the interaction strength $V / J$ for a lattice with $I=N=12$. The solid line shows the condensate $q=0$ state, the dashed and dotted lines correspond to the first and second excited quasimomentum state, respectively.

\section{B. Superfluid fraction}

One of the most interesting physical quantities in an interacting many-boson system is the superfluid density. It is the natural order parameter for the various superfluid to insulator phase transitions, e.g., the superfluid to Mott-insulator transition observed experimentally [1].

\section{Definition}

The macroscopic, as well as the microscopic definition of superfluidity, are connected to flow properties. Macroscopically, one usually employs the two-fluid picture to describe systems which exhibit superfluidity. The superfluid and the normal fluid are distinguished through their behavior in the presence of moving boundaries. For example, in a narrow channel with moving side walls only the normal fluid is dragged along with the walls, whereas the superfluid stays at rest. In the rest frame of the walls only the superfluid moves. From the difference between the energies (in the rest frame of the walls) of a system with moving and one with stationary walls, one can directly extract the kinetic energy of the superfluid component and hence, via its velocity, the superfluid density [26, 27].

A microscopic definition of superfluidity can be constructed in analogy to this simple macroscopic picture. First of all we consider the generalized condensate wave function $\chi(\vec{x})=\mathrm{e}^{\mathrm{i} \vartheta(\vec{x})}|\chi(\vec{x})|$ as defined in the previous section. A spatial variation of the phase $\vartheta(\vec{x})$ of the condensate wave function is connected to a velocity field [28]

$$
\vec{v}_{\mathrm{s}}=\frac{\hbar}{m} \vec{\nabla} \vartheta(\vec{x})
$$

The flow described by this velocity field is non-dissipative and irrotational, properties which conform with the 
macroscopic picture of superfluidity. This velocity is therefore identified with the flow field of a superfluid 28. In principle Eq. (16) provides the basic microscopic distinction of the superfluid through its flow behavior.

As in the macroscopic case we can develop an explicit expression for the superfluid fraction by considering the total energy of the system. For simplicity we restrict ourselves to a one-dimensional system. Assume that we impose a linear phase variation with a total phase twist $\Theta$ over the length $L$ of the system, i.e., $\vartheta(x)=\Theta x / L$. One way to accomplished this is by imposing twisted boundary conditions on the many-body wave function

$$
\Psi\left(x_{1}, \ldots, x_{k}+L, \ldots, x_{N}\right)=\mathrm{e}^{\mathrm{i} \Theta} \Psi\left(x_{1}, \ldots, x_{k}, \ldots, x_{N}\right)
$$

for all $k$ 29, 30, 31]. This imposed phase gradient generates a flow with a velocity $v_{\mathrm{s}}=\hbar \Theta /(m L)$. The portion of the system which responds to the phase gradient by flowing with this velocity is called superfluid. The presence of the flow will increase the total energy of the system. As long as other excitations are absent, that is, as long as the imposed phase gradient is small, the increase in the total energy can be attributed solely to the kinetic energy of the superflow

$$
E_{\Theta}-E_{0}=\frac{1}{2} M_{\mathrm{s}} v_{\mathrm{s}}^{2} .
$$

Here $E_{0}$ is the ground state energy of the system without phase twist and $E_{\Theta}$ is the ground state energy obtained with twisted boundary conditions. The mass $M_{\mathrm{s}}$ corresponds to the total mass of the superfluid portion, by introducing the superfluid fraction $f_{\mathrm{s}}$ is can be rewritten as $M_{\mathrm{s}}=m N f_{\mathrm{s}}$. From Eq. (18) we can extract a simple expression for the superfluid fraction [29, 32, 33.

$$
f_{\mathrm{s}}=\frac{2 m L^{2}}{\hbar^{2} N} \frac{E_{\Theta}-E_{0}}{\Theta^{2}} \quad \text { for } \Theta \ll \pi .
$$

Hence the superfluid fraction is determined by the stiffness of the system under phase variations. We have to stress that the twist angle $\Theta$ has to be sufficiently small to avoid effects other than the collective flow of the superfluid component. We will return to this point at the end of this section.

The definition of the superfluid fraction through the energy change under imposed phase variations corresponds to the so-called helicity modulus introduced in Ref. 29]. Moreover, it is equivalent to the concept of winding numbers which is used in path-integral Monte Carlo methods [26]. All these measures predict the noninteracting Bose gas to be a perfect superfluid. This is in contradiction to the Landau picture of superfluidity which is based on the dispersion relation of elementary excitations which defines a critical velocity. In the Landau picture the ideal Bose gas is not considered a superfluid since its critical velocity is zero. A possible connection between these two pictures of superfluidity is still a matter of debate 27]. We adopt a pragmatic view and use the definition (19) keeping in mind that it does not tell anything about the dynamical stability of the superfluid flow at finite velocities.

A second comment is appropriate concerning the relation between superfluidity and Bose-Einstein condensation. First of all one should realize that although the phase of the condensate wave function determines the superfluid velocity (16), the superfluid and the condensate fraction are not the same. A famous example is liquid ${ }^{4} \mathrm{He}$ at zero temperature which is $100 \%$ superfluid but only $10 \%$ of the atoms are in the condensate [28]. From the formal point of view we used the condensate wave function only to motivate the connection between twisted boundary conditions (17) and the superfluid flow. On the level of the resulting Eqs. (17) and (19) the condensate does not appear. In general it is not strictly evident that superfluidity presupposes the presence of a condensate [27.

The relation (19) for the continuous system can be easily transfered to the Bose-Hubbard model. Basically one replaces the length $L$ of the continuous system by the number of lattice sites $I$ and the prefactor of the kinetic energy $\hbar^{2} /(2 m)$ by the tunneling strength $J$ of the BoseHubbard Hamiltonian. The superfluid fraction for the discrete lattice thus reads [5]

$$
f_{\mathrm{s}}=\frac{I^{2}}{J N} \frac{E_{\Theta}-E_{0}}{\Theta^{2}} \quad \text { for } \Theta \ll \pi .
$$

In order to compute the ground state energy of a Bose-Hubbard system with imposed phase gradient, the use of twisted boundary conditions is impracticable. By means of the local unitary transformation $\hat{\mathrm{U}}_{\Theta}=$ $\prod_{k=1}^{N} \exp \left(\mathrm{i} \Theta \hat{\mathrm{x}}_{k} / L\right)$ we can map the phase twist from the state onto the Hamiltonian. The eigenvalues of the resulting twisted Hamiltonian for periodic boundary conditions are identical to those of the original Hamiltonian with twisted boundary conditions. The twisted BoseHubbard Hamiltonian reads

$$
\begin{aligned}
\hat{\mathrm{H}}_{\Theta}= & -J \sum_{i=1}^{I}\left(\mathrm{e}^{-\mathrm{i} \Theta / I} \hat{\mathrm{a}}_{i+1}^{\dagger} \hat{\mathrm{a}}_{i}+\text { h.a. }\right) \\
& +\sum_{i=1}^{I} \epsilon_{i} \hat{\mathrm{n}}_{i}+\frac{V}{2} \sum_{i=1}^{I} \hat{\mathrm{n}}_{i}\left(\hat{\mathrm{n}}_{i}-1\right) .
\end{aligned}
$$

Compared to the original Hamiltonian (3) only the offdiagonal hopping term is modified by an additional factor $\mathrm{e}^{\mp \mathrm{i} \Theta / I}$, the so-called Peierls phase factor 30, 31]. Physically this term ensures that any particle which tunnels to an adjacent site acquires the correct phase in order to establish the linear phase variation across the system.

There are several ways to impose this phase factor experimentally. One possibility is to add a linear external potential or a homogeneous electric field in the case of charged particles. Another possibility is a constant acceleration of the lattice, which in the case of optical standing wave lattices can be realized by ramping the detuning between the two counter-propagating beams. One might 


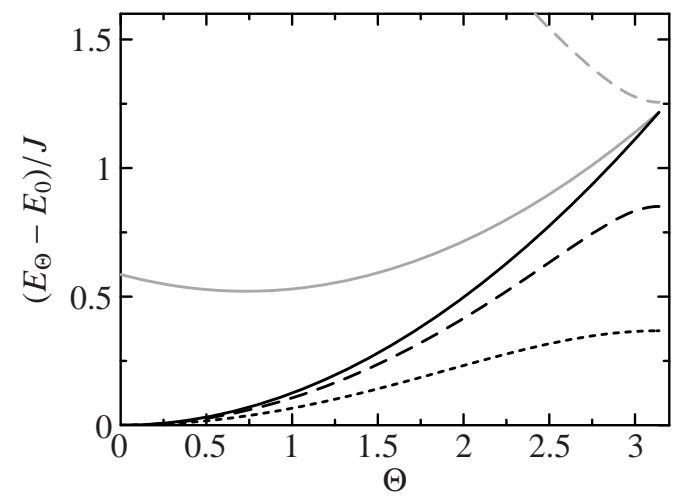

FIG. 5: Difference $E_{\Theta}-E_{0}$ between the ground state energies with an imposed phase twist and without as function of the total twist angle $\Theta$ for a system with $I=N=8$. The different curves correspond to $V / J=0$ (solid line), $V / J=3$ (dashed), and $V / J=5$ (dotted). The gray lines depict the energy difference for the first excited state of the twisted system.

be able to utilize this possibility to probe superfluidity directly.

A straightforward procedure to compute the superfluid fraction is now evident: We numerically solve the eigenvalue problems of the twisted and the non-twisted Hamiltonian, $\hat{\mathrm{H}}_{\Theta}$ and $\hat{\mathrm{H}}_{0}$, with periodic boundary conditions and insert the resulting ground state energies $E_{\Theta}$ and $E_{0}$ into (20).

Before doing so, it is useful to study how the energy difference $E_{\Theta}-E_{0}$ entering into Eq. (20) depends on the twist angle $\Theta$. We have already assumed that $\Theta$ has to be sufficiently small to avoid excitations other than the collective flow of the superfluid component. Figure [5 depicts the result of a numerical calculation for $E_{\Theta}-E_{0}$ as function of $\Theta$ for different values of $V / J$. For the noninteracting system (black solid curve) the energy is proportional to $\Theta^{2}$ up to large values of $\Theta$, i.e., the superfluid fraction is independent of the actual value of $\Theta$ used in the calculation. However, one should note that at $\Theta=\pi$, which corresponds to antiperiodic boundary conditions, a level crossing between the ground state and the first excited state of the twisted system appears. The presence of interactions leads to a "level repulsion" between these two states (see dashed and dotted curves in Fig. 5). Hence the energy deviates significantly from the $E_{\Theta}-E_{0} \propto \Theta^{2}$ behavior for $\Theta \sim \pi$. If one were to use Eq. (20) for $\Theta \sim \pi$ one would underestimate the superfluid fraction significantly. A physically meaningful application of Eq. (20) to determine the superfluid fraction therefore requires $\Theta \ll \pi$. Formally one might even impose the limit $\Theta \rightarrow 0$. For all the following numerical calculations we will use $\Theta=0.1$.

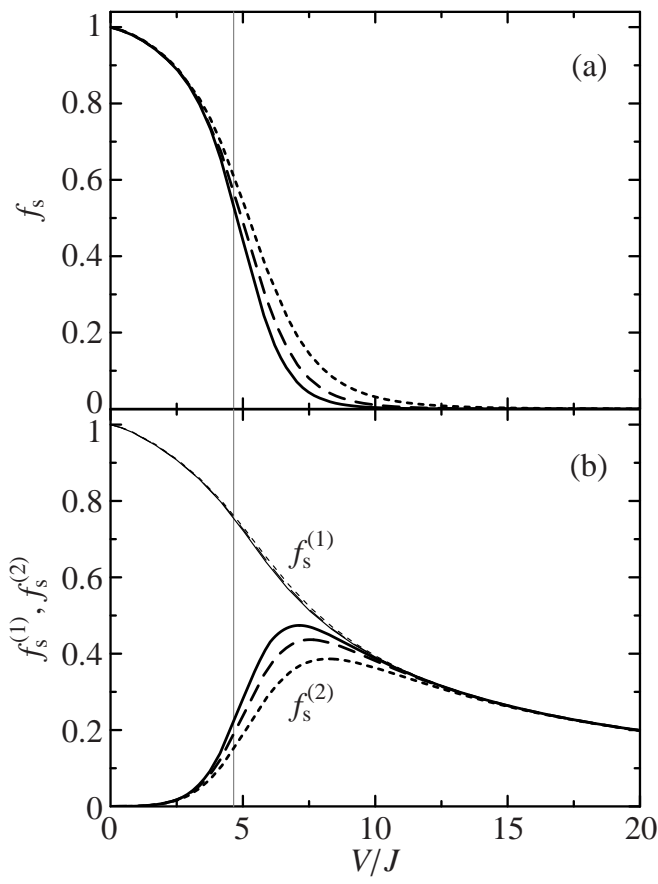

FIG. 6: (a) Superfluid fraction $f_{\mathrm{s}}$ for one-dimensional BoseHubbard systems with $I=N=12$ (solid), 10 (dashed), and 8 (dotted) as function of the interaction strength $V / J$. (b) First and second order contribution to $f_{\mathrm{s}}$ in the perturbative calculation. The vertical gray line marks the critical interaction strength $(V / J)_{\mathrm{cr}}=4.65$ for the Mott-insulator transition in an infinite system 14, 19].

\section{Direct numerical calculation}

We now employ Eq. (20) to compute the superfluid fraction $f_{\mathrm{s}}$ from the energies $E_{\Theta}$ and $E_{0}$ obtained numerically. The results for systems with commensurate filling $N / I=1$ and lattice sizes up to $I=12$ are shown in Fig. 6(a).

In the range $3<V / J<6$ the superfluid fraction, i.e. the order parameter for the superfluid to Mott-insulator transition, declines rapidly with increasing interaction strength $V / J$. In these relatively small systems we already observe a rapid transition from the superfluid phase at small $V / J$ to an insulating phase with vanishing superfluid fraction at large $V / J$. The transition region is in good agreement with the critical point $(V / J)_{\mathrm{cr}}=4.65$ for the infinite lattice determined by Monte Carlo simulations [14] or a strong coupling expansion [19].

The dependence of the superfluid fraction on the lattice size shown in Fig. 6(a) is rather moderate. In the superfluid phase for up to $V / J \leq 4$ there is no noticeable size dependence. Only the shoulder of the curve exhibits a size dependence: With increasing $I$ the decrease in $f_{\mathrm{s}}$ steepens and the tail of the curve is compressed and shifted towards lower $V / J$. From this observation we conclude that systems with $I \approx 10$ already allow very good qualitative, and even semi-quantitative conclusions, about the superfluid behavior of larger systems. 
The rapid decrease of the superfluid fraction is in stark contrast to the behavior of the number fluctuations shown in Fig. 1(a). Evidently, the number fluctuations still exhibit values of up to $\sigma_{i} \approx 0.3$ in cases where the superfluid fraction has already vanished. This proves that the Mott-insulator state can still be a superposition of many Fock states and thus show considerable number fluctuations. Only in the limit $V / J \rightarrow \infty$ when $\sigma_{i}$ vanishes it becomes a pure Fock state.

\section{Perturbative treatment}

Apart from the direct solution of the eigenvalue problems of the twisted and the non-twisted Hamiltonian one can use a perturbative approach to determine the energy difference $E_{\Theta}-E_{0}$. As the superfluid fraction (20) is defined only for very small twist angles $\Theta$ this perturbative treatment does not introduce any additional approximation. All terms of the expansion that contribute in the limit $\Theta \rightarrow 0$ will be included.

The derivation consists of two steps: We first expand the twisted Hamiltonian in a power series up to second order in $\Theta$

$$
\hat{\mathrm{H}}_{\Theta} \simeq \hat{\mathrm{H}}_{0}+\frac{\Theta}{I} \hat{\mathrm{J}}-\frac{\Theta^{2}}{2 I^{2}} \hat{\mathrm{T}}=\hat{\mathrm{H}}_{0}+\hat{\mathrm{H}}_{\text {pert }},
$$

where the current operator $\hat{J}$ and the kinetic energy operator $\hat{\mathrm{T}}$ are given by:

$$
\begin{aligned}
& \hat{\mathrm{J}}=\mathrm{i} J \sum_{i=1}^{I}\left(\hat{\mathrm{a}}_{i+1}^{\dagger} \hat{\mathrm{a}}_{i}-\text { h.a. }\right) \\
& \hat{\mathrm{T}}=-J \sum_{i=1}^{I}\left(\hat{\mathrm{a}}_{i+1}^{\dagger} \hat{\mathrm{a}}_{i}+\text { h.a. }\right) .
\end{aligned}
$$

We then compute the energy shift $E_{\Theta}-E_{0}$ caused by the perturbation $\hat{\mathrm{H}}_{\text {pert }}$ in (22) via second order perturbation theory. The lowest order contributions to the energy shift are quadratic in $\Theta$. Inserting them into (20) leads to an expression for the superfluid fraction consisting of two terms

$$
f_{\mathrm{s}}=f_{\mathrm{s}}^{(1)}-f_{\mathrm{s}}^{(2)} .
$$

The first order term is proportional to the kinetic energy expectation value

$$
f_{\mathrm{s}}^{(1)}=-\frac{1}{2 N J}\left\langle\Psi_{0}|\hat{\mathrm{T}}| \Psi_{0}\right\rangle=\frac{1}{N} \sum_{i=1}^{I} \rho_{i, i+1}^{(1)},
$$

where $\Psi_{0}$ is the ground state of the original Hamiltonian $\hat{\mathrm{H}}_{0}$ and $\rho_{i j}^{(1)}$ the associated one-body density matrix introduced in Eq. (10). The second order term involves matrix elements of the current operator between the ground state $\left|\Psi_{0}\right\rangle$ and all the excited states $\left|\Psi_{\nu}\right\rangle(\nu=1,2, \ldots)$ of the original Hamiltonian $\hat{\mathrm{H}}_{0}$,

$$
f_{\mathrm{s}}^{(2)}=\frac{1}{N J} \sum_{\nu \neq 0} \frac{\left|\left\langle\Psi_{\nu}|\hat{J}| \Psi_{0}\right\rangle\right|^{2}}{E^{(\nu)}-E^{(0)}} .
$$

All higher-order terms of the perturbative expansion of the energy shift do not contribute to the superfluid fraction in the limit $\Theta \rightarrow 0$. Thus Eq. (24) is an exact expression for $f_{\mathrm{s}}$.

We note that this derivation and the resulting expression is closely related to the so-called Drude weight which characterizes the dc conductivity of charged fermionic systems [34.

This formulation of the superfluid fraction provides a detailed insight into the mechanisms that govern the superfluid properties of the system. First of all it shows that superfluidity is not a property of the ground state, but rather the response of the system to an external perturbation. The second order term (26) introduces an explicit dependence on the whole excitation spectrum. Since this second order contribution always lowers the total superfluid fraction we can conclude that the coupling to excited states leads to a suppression of superfluidity. The first order term (25), which is just the ground state expectation value of the kinetic energy and can be expressed in terms of the one-body density matrix of the ground state alone, gives only an upper bound for $f_{\mathrm{s}} 35$.

The behavior of the first and second order contributions to the superfluid fraction for a regular lattice are shown in Fig. 6(b). The first order term (25), i.e. the rescaled kinetic energy expectation value, is approximately one for small $V / J$ and decreases slowly with increasing interaction strength. Within the insulating phase, where the total superfluid fraction shown in Fig. [6) (a) has vanished completely, the first order contribution $f_{\mathrm{s}}^{(1)}$ still has values of up to 0.3 .

The vanishing of the total superfluid fraction is caused by a characteristic behavior of the second order contribution (26): In the transition region $f_{\mathrm{s}}^{(2)}$ shows a thresholdlike increase from zero to its maximum value. For values of $V / J$ beyond this maximum the second order term quickly converges to the value of $f_{\mathrm{s}}^{(1)}$. Thus the vanishing of the superfluid fraction in insulating phases, e.g., the Mott-insulator, results from the cancellation between first and second order term which both have considerable size.

This clearly demonstrates that the coupling to the excited states and the structure of the excitation spectrum is essential for the superfluid properties of the system. The ground state alone, represented by the first order contribution $f_{\mathrm{s}}^{(1)}$, provides only limited informationnamely an upper bound - on the superfluid fraction. Therefore, any observable which is sensitive to the ground state only, such as number fluctuations, condensate fraction, and interference pattern, cannot provide clear information the superfluid properties of the system.

The only other observable which is sensitive to the properties of the excitation spectrum is the energy gap 
$E_{\text {gap }}$ between the ground state and the first excited state. As we have shown in Sec. $1 \mathrm{C}$ the energy gap starts to grow linearly with $V / J$ in the Mott-insulator phase. This is another indicator for the change in the excitation spectrum that also leads to the vanishing of the superfluid fraction. However, as Eq. (26) illustrates, the dependence of the superfluid fraction on the excited states is more complex than just the energy gap.

\section{EXPERIMENTAL OBSERVABLES}

The two quantities, condensate fraction and superfluid fraction, discussed in the preceeding section describe two important and fundamental properties of the system, however, they are not directly accessible to simple experiments. In this section we discuss two observables which are directly accessible to present day experiments and show their relation to the fundamental quantities discussed above.

\section{A. Matter-wave interference pattern}

The simplest experimental procedure to obtain information on the state of the Bose gas in the lattice is to release the atoms from the lattice and detect the matter-wave interference pattern after some time of flight [1, 2, 9]. The crucial question is, how much the interference pattern can tell us about the superfluid or the condensed portion of the system.

Neglecting interactions during the expansion of the gas we can describe the time evolution of the single-particle states, which are initially localized at the different lattice sites, by Gaussian wave-packets. The matter-wave intensity observed at a point $\vec{y}$ after ballistic expansion for a time $\tau$ can be written as [5]

$$
\mathcal{I}(\vec{y})=\left\langle\Psi_{0}\left|\hat{\mathrm{A}}^{\dagger}(\vec{y}) \hat{\mathrm{A}}(\vec{y})\right| \Psi_{0}\right\rangle
$$

The amplitude operators are given by

$$
\hat{\mathrm{A}}(\vec{y})=\sum_{i=1}^{I} G_{i}(\vec{y}, \tau) \hat{\mathrm{a}}_{i},
$$

where $G_{i}(\vec{y}, \tau)$ is the amplitude of a Gaussian wavepacket that originates from site $i$ after free expansion for a time $\tau$. We are interested only in the generic structure of the intensity pattern, i.e., the presence or the absence of interference peaks. Therefore, we can simplify the treatment significantly by dropping all the terms related to the spatial envelope of the interference pattern. The Gaussian can thus be replaced by a phase factor and the amplitude operator reduces to

$$
\hat{\mathrm{A}}(\vec{y})=\sum_{i=1}^{I} \mathrm{e}^{\mathrm{i} \phi_{i}(\vec{y}, \tau)} \hat{\mathrm{a}}_{i}=\sum_{i=1}^{I} \mathrm{e}^{\mathrm{i} \delta \phi(\vec{y}, \tau) i} \hat{\mathrm{a}}_{i},
$$

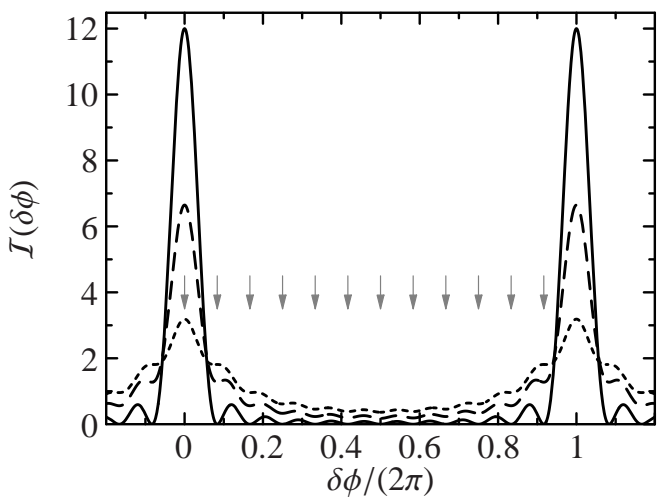

FIG. 7: Matter-wave intensity as function of the phase difference $\delta \phi$ for a one-dimensional lattice system with $I=N=12$ and different interaction strengths $V / J=0$ (solid line), 5 (dashed), and 8 (dotted).

Here $\phi_{i}(\vec{y}, \tau)$ is the phase accumulated on the path from the $i$ th site to the observation point $\vec{y}$. In the far-field approximation this can be replaced by $\delta \phi(\vec{y}, \tau) i$, where $\delta \phi(\vec{y}, \tau)$ is the phase difference between paths originating from adjacent lattice sites. The matter-wave intensity (27) as function of $\delta \phi$ now reads

$$
\begin{aligned}
\mathcal{I}(\delta \phi) & =\frac{1}{I} \sum_{i, j=1}^{I} \mathrm{e}^{\mathrm{i} \delta \phi(i-j)}\left\langle\Psi_{0}\left|\hat{\mathrm{a}}_{j}^{\dagger} \hat{\mathrm{a}}_{i}\right| \Psi_{0}\right\rangle \\
& =\frac{1}{I} \sum_{i, j=1}^{I} \mathrm{e}^{\mathrm{i} \delta \phi(i-j)} \rho_{i j}^{(1)} .
\end{aligned}
$$

This result proves the direct relation between the interference pattern and the quasimomentum distribution of the gas in the lattice given by Eq. (15) 5]. The occupation number $\tilde{n}_{q}$ of the quasimomentum $q$ state is given by the intensity $\mathcal{I}(\delta \phi)$ for a phase difference $\delta \phi=q a$ :

$$
\tilde{n}_{q}=\mathcal{I}(\delta \phi=q a) .
$$

As a special case of this result, the condensate fraction, i.e. the occupation of the $q=0$ state, is proportional to the intensity for $\delta \phi=0$. Of course, this result agrees with the naive view that the interference pattern simply gives the momentum distribution of the trapped gas 15$]$.

Furthermore Eq. (30) enables us to draw an important conclusion about the relation between the matter-wave interference pattern and the superfluid properties of the system. The interference pattern depends exclusively on the one-body density matrix $\rho_{i j}^{(1)}$ for the ground state. The superfluid fraction, in contrast, depends crucially on the excited states of the system as we have discussed in detail in Sec. IIIB3 Thus the interference pattern cannot give complete information on the superfluid properties of the system. It does not probe the physics that is crucial for superfluidity.

The matter-wave interference patterns which result from Eq. (30) for three different interaction strength 
$V / J$ are depicted in Fig. 7 For the noninteracting system (solid curve) it exhibits sharp interference peaks at $\delta \phi=0$ and $2 \pi$ which correspond to the principal peaks observed experimentally [1]. Because we discarded the terms related to the envelope, the intensity $\mathcal{I}(\delta \phi)$ is simply a $2 \pi$-periodic function in $\delta \phi$. With increasing interaction strength $V / J$ (dashed and dotted curves in Fig. 17) the amplitude of the principal peaks is reduced and an "incoherent background" emerges. Effectively this leads to a gradual broadening of the interference peaks which was observed experimentally [1].

The emergence of the background has a straightforward interpretation in terms of the quasimomentum distribution. The gray arrows in Fig. 7 mark the values of $\delta \phi$ which correspond to the discrete quasimomenta $q$ allowed in the lattice. According to (31) the intensity at these points just gives the occupation numbers for the different quasimomentum states. Thus the depletion of the principal peak with increasing $V / J$ just indicates the depletion of the condensate. The emergence of a background shows that states with nonvanishing quasimomenta are successively populated - as we have already discussed in Sec. IIIA2 In the limit of strong interactions all quasimomentum states of the band are occupied uniformly and the corresponding interference pattern is perfectly flat.

The fact that the vanishing of the superfluid fraction is not associated with the vanishing of the interference fringes is highlighted by these numerical results. The values of $V / J$ used to compute the three intensity distributions in Fig. 7 correspond to superfluid fractions of $f_{\mathrm{s}}=1$ (solid curve), $f_{\mathrm{s}} \approx 0.5$ (dashed), and $f_{\mathrm{s}} \approx 0$ (dotted). In the latter case, interference fringes are still clearly visible although the system is a perfect insulator, i.e., the superfluid fraction is zero. Thus, we see that with increasing $V / J$ the superfluid component vanishes much earlier than the interference fringes. In other words, the Mott insulator phase still exhibits a degree of phase coherence.

As a simple quantity to characterize the interference pattern one might introduce the visibility of the fringes. The standard definition of the fringe visibility

$$
\mathcal{V}=\frac{\mathcal{I}_{\max }-\mathcal{I}_{\min }}{\mathcal{I}_{\max }+\mathcal{I}_{\min }}
$$

only relies on the maximum and minimum values of the intensity. For a regular lattice with even number of sites we immediately find $\mathcal{I}_{\max }=\mathcal{I}(0)$ and $\mathcal{I}_{\min }=\mathcal{I}(\pi)$. On the basis of (31) we can identify the maximum (minimum) intensity with the largest (smallest) quasimomentum occupation number. This definition of the visibility therefore provides a direct measure for the inhomogeneity of the quasimomentum distribution: $\mathcal{V}$ is 1 as long as there are unoccupied quasimomentum states and it goes to zero if the band is filled uniformly.

The dependence of the visibility measure on the interaction strength is depicted in Fig. 8. The visibility decreases only very slowly with increasing $V / J$ and can

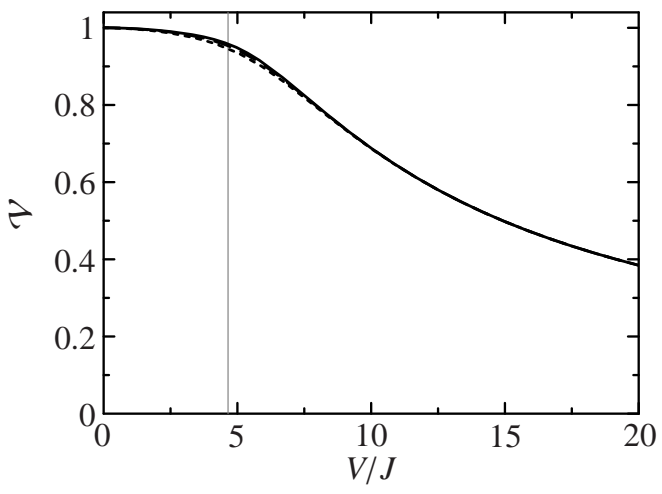

FIG. 8: Visibility $\mathcal{V}$ of the interference fringes as function of $V / J$ for lattices with commensurate filling $N / I=1$ and $\mathrm{I}=12$ (solid), $\mathrm{I}=10$ (dashed), and $I=8$ (dotted) lattice sites.

have values up to $\mathcal{V} \approx 0.7$ in the insulating phase where $f_{\mathrm{s}} \approx 0$. This confirms the observation that superfluidity vanishes much faster than the interference fringes and thus the coherence of the system [5]. The fact that the curves in Fig. [ 8 are practically independent of the lattice size proves that this statement also holds for large lattices and is not an artifact of the relatively limited size. With regard to the quasimomentum distribution the slow decrease of $\mathcal{V}$ indicates that very large ratios $V / J$ are required to achieve a perfectly uniform occupation of the band.

One can define other measures for the fringe visibility which are more sensitive to particular changes in the interference patterns. However, the most important and decisive measure for the coherence properties of the system is the interference pattern itself.

\section{B. Structure factor}

In the presence of a periodic modulation of the lattice potential the atom density distribution will develop a spatial structure [8]. Systems of this type will be discussed in detail in Sec. D These periodic density modulations should be observable using Bragg diffraction of light from the trapped atomic gas. Experiments of this kind have already been performed using laser-cooled atomic gases in optical lattices [36, 37].

The quantity directly accessible through Bragg diffraction experiments is the structure factor $S(k)$ which is given by 38

$$
S(k)=\frac{1}{I^{2}} \sum_{i, j=1}^{I} \mathrm{e}^{\mathrm{i} k\left(\xi_{i}-\xi_{j}\right)}\left\langle\Psi_{0}\left|\hat{\mathrm{n}}_{i} \hat{\mathrm{n}}_{j}\right| \Psi_{0}\right\rangle .
$$

This quantity is sensitive to density-density correlations and is therefore a suitable indicator for the presence of diagonal long-range order. For a homogeneous occupation of all lattice sites, present in the regular lattices discussed so far, the structure factor exhibits distinct peaks at integer values of $k a /(2 \pi)$. The presence of any additional 
periodic structure in the atomic density distribution will lead to the appearance of new peaks at noninteger values of $k a /(2 \pi)$. These signal the presence of diagonal long-range order. From the position and the relative amplitude of the additional peaks in the structure factor one can derive detailed information on the spatial density distribution. We will discuss this in detail in Sec. [VC]

\section{TWO-COLOR SUPERLATTICES}

Having discussed the relevant observables on a formal level and compared their behavior for the superfluid to Mott-insulator transition in a regular lattice we now want to explore another aspect of the phase diagram of the zero temperature Bose gas. Optical lattices offer the unique possibility of modifying the topology of the lattice in a flexible and perfectly controlled manner. They thus open new possibilities for studying many-body physics in nonuniform lattice potentials. In contrast to most solid-state systems, where the precise topology of the irregular lattice is difficult to specify, the high degree of experimental control facilitates investigations into specific aspects of "disorder"-induced phenomena.

\section{A. Optical two-color lattices}

The simplest way to experimentally generate a nonuniform lattice potential is through a superposition of two collinear optical standing waves with different wavelengths - a so called two-color lattice. The interference between these standing waves produces a superlattice with a sinusoidal modulation of the depth of the lattice wells. More complex topologies have already been realized experimentally by using a superposition of several laser beams with different wave vectors [39]. Truly random lattices can be generated by superimposing a speckle pattern 13. Thus optical lattices cover the whole range from spatially modulated lattice potentials to disordered systems.

In the following we consider a superlattice generated by the interference of two standing waves with wavelengths $\lambda_{1}$ and $\lambda_{2}=\frac{5}{7} \lambda_{1}$. The intensity of the second laser field is assumed to be much smaller than the intensity of the first laser, in this example $I_{2}=0.04 I_{1}$. This combination results in a superlattice consisting of identical unit cells each composed of 5 elementary lattice sites. Figure 9(a) depicts the lattice potential $U_{\text {lat }}(x)$ for a certain value of the relative phase.

In the language of the Bose-Hubbard model this spatial modulation of the well depths corresponds to a modulation of the on-site energies $\epsilon_{i}$. We assume a sinusoidal variation of the $\epsilon_{i}$ in the range $[-\Delta, 0]$ as depicted in Fig. 9 (b). The parameter $\Delta$ characterizes the amplitude of the modulation, for $\Delta=0$ we recover the regular lattice potential. The modulation of the lattice poten-
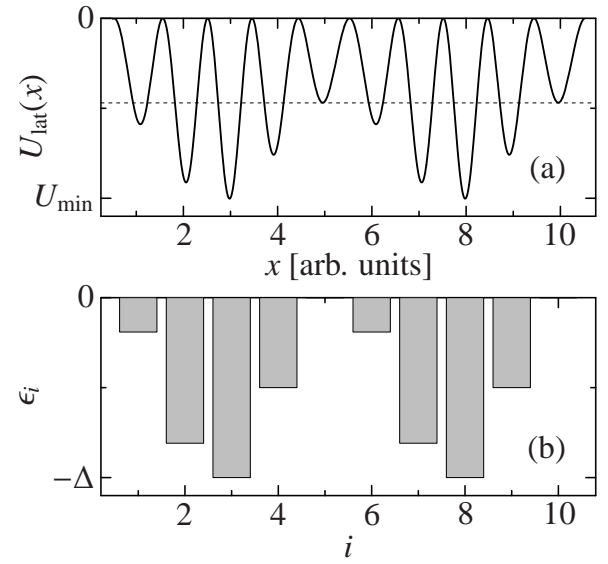

FIG. 9: (a) Lattice potential generated by superposition of two optical standing waves (see text). (b) Corresponding distribution of the on-site energies $\epsilon_{i}$ with modulation amplitude $\Delta$ (after subtraction of an over-all constant).

tial also influences the tunneling coefficient $J$ which, in general, varies for the different lattice sites. For the lattices considered here the influence of this variation can be neglected in good approximation 13.

\section{B. Localized phase}

As a first step we consider a noninteracting Bose gas in the superlattice depicted in Fig. 9] We solve the eigenvalue problem for the Bose-Hubbard Hamiltonian including two unit cells with filling factor 1 , i.e., $I=N=10$. For $V / J=0$ we successively increase the amplitude $\Delta$ of the spatial modulation of the on-site energies. The resulting distributions of mean occupation numbers $\bar{n}_{i}$ and number fluctuations $\sigma_{i}$ for the individual sites are depicted in Fig. 10] For a regular system, without any modulation of the $\epsilon_{i}$, the lattice is occupied homogeneously. With increasing modulation amplitude $\Delta / J$ the particles preferentially occupy those lattice sites with lower $\epsilon_{i}$ as one would classically expect. The degree of localization is governed by the competition between the on-site energies and the tunneling term of the Bose-Hubbard Hamiltonian (3). For sufficiently large $\Delta / J$ only the site with the lowest $\epsilon_{i}$ in each unit cell is populated. The number fluctuations $\sigma_{i}$ behave accordingly: with increasing $\Delta / J$ the number fluctuations at the deepest lattice well grow whereas the fluctuations at all other wells slowly decrease. In the limit of large $\Delta / J$ the ground state is a superposition of all Fock states which have nonzero occupation numbers only at the deepest well of each unit cell.

There are several possibilities for detecting this localized phase in an experiment. The simplest signature appears in the interference pattern after the expansion of the gas. Figure 11 shows the intensity distribution that results from Eq. (30) for three different values of $\Delta / J$. 


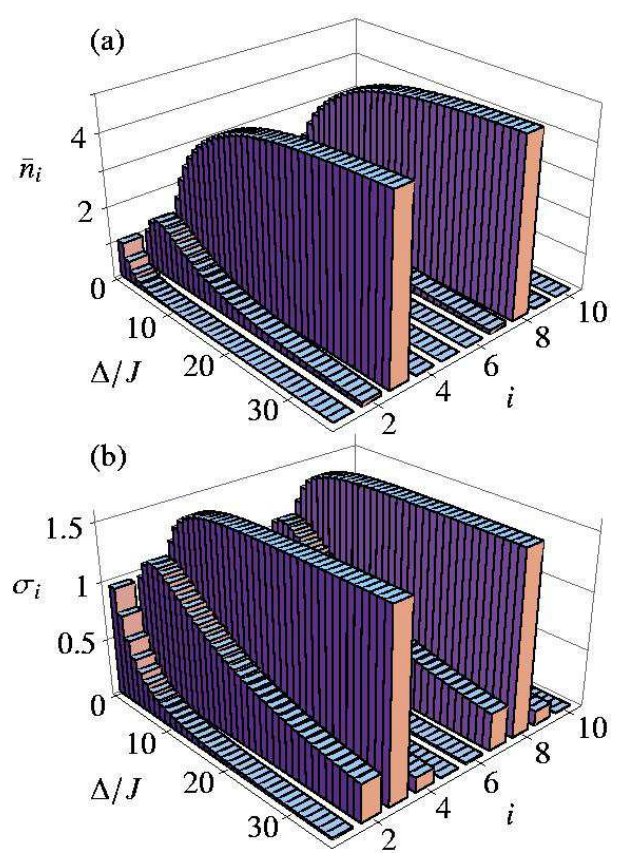

FIG. 10: Mean occupation numbers $\bar{n}_{i}$ (a) and number fluctuations $\sigma_{i}$ (b) for the different lattice sites $i=1, \ldots, 10$ as function of the amplitude $\Delta / J$ of the sinusoidal modulation for the noninteracting gas.

In the absence of the spatial modulation (upper panel) we observe the interference pattern of a regular lattice discussed in Sec. IVA With increasing $\Delta / J$, that is increasing degree of localization, additional peaks emerge. First the peaks next to initial principal peaks appear, then the next-order peaks emerge. In the limit of perfect localization we obtain a regular interference pattern with a peak separation corresponding to the inverse of the number of elementary sites in a unit cell. In this manner the relative strength of the emerging peaks provides a direct experimental measure for the degree of localization in these superlattices.

Another experimental observable that is sensitive to the degree of localization is the structure factor $S(k a)$ introduced in Sec. IVB We will come back to this quantity in the next section.

\section{Quasi Bose-glass phase}

It is intuitively clear that repulsive interactions will have a strong influence on the localized phase: A concentration of many particles at a single lattice site will generate a large interaction energy contribution. Thus the interaction enforces delocalization of the particles.

To illustrate the competition between lattice modulation and repulsive two-body interaction Fig. 12 depicts the change of the mean occupation numbers $\bar{n}_{i}$ and the number fluctuations $\sigma_{i}$ with increasing interaction strength $V / J$ for a fixed modulation amplitude

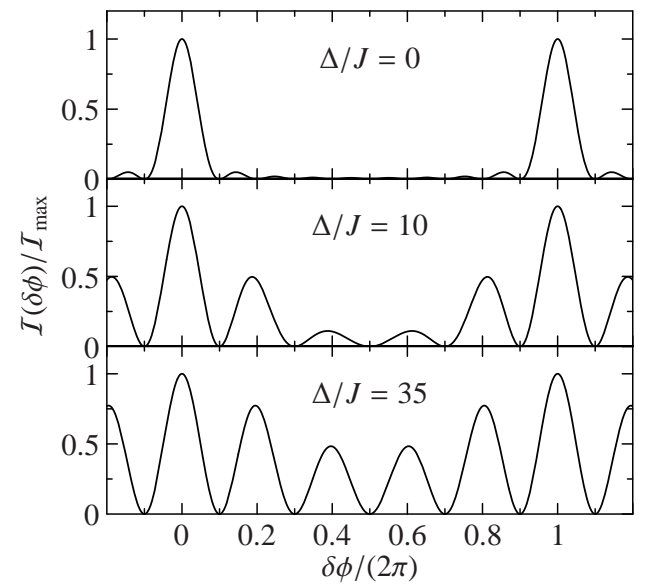

FIG. 11: Interference pattern after release from the lattice for different values of the amplitude $\Delta / J$ of the sinusoidal modulation of the on-site energies.

$\Delta / J=50$.

The perfectly localized configuration persists only at very small interaction strengths. Already for $V / J \gtrsim 2$ it is energetically favorable to reduce the maximum occupation number and thus the interaction energy by redistributing particles to sites with higher on-site energies $\epsilon_{i}$. This region of interaction-induced delocalization is sometimes termed Anderson-glass phase [6, 13]. Initially the changes in the mean occupation numbers happen continuously. However, for $V / J \gtrsim 10$ this behavior changes. As shown in Fig. 12(a) there are extended intervals of interaction strengths where the mean occupation numbers are constant and approximately integer. Between these regions of "stable" configurations there are narrow windows in which some of the $\bar{n}_{i}$ change by roughly \pm 1 . In the present example one of these almost steplike rearrangements happens at $V / J \approx 30$. This behavior of the mean occupation numbers is also reflected in the number fluctuations depicted in Fig. 12(b). Within the stable regions all $\sigma_{i}$ are rather small, i.e., the dominant contribution to the expansion (5) of the ground state comes from a single Fock state. The steplike rearrangements are accompanied by an increase of the number fluctuations of those sites that change their mean occupation number.

It is the subtle interplay between interaction and lattice irregularity that drives these successive rearrangements between configurations with integer occupation numbers. The sequence of rearrangements depends crucially on the topology of the lattice, i.e., the set of on-site energies. If the distribution of the $\epsilon_{i}$ becomes more homogeneous (meaning that the number of different values of the $\epsilon_{i}$ increases) then the number of possible rearrangements increases and the stable regions shrink. In the limit of a lattice with random disorder this leads to the so-called Bose-glass phase [6, 7]. For the superlattices with a rather small number of different on-site energies we will use the term quasi Bose-glass phase. The study of the superfluid fraction in Sec. $\mathrm{VD}$ shows that the quasi 

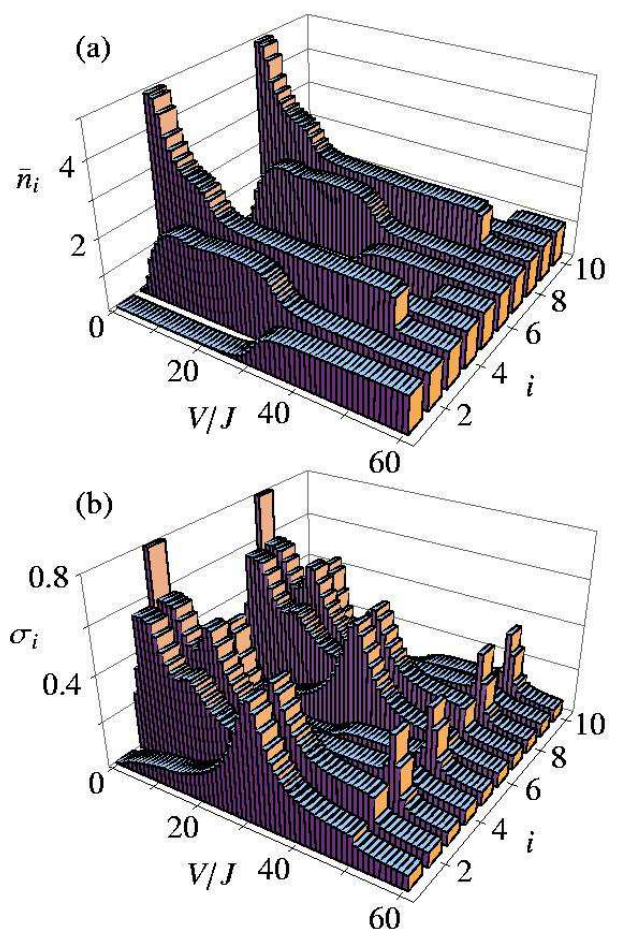

FIG. 12: Mean occupation numbers $\bar{n}_{i}$ (a) and the number fluctuations $\sigma_{i}$ (b) for the individual lattice sites $i=1, \ldots, 10$ as function of the interaction strength $V / J$ for fixed modulation amplitude $\Delta / J=50$.

Bose-glass phase is insulating and that the energy gap is small.

If the interaction strength reaches a value $V=\Delta$ a final and very sharp rearrangement happens and the system assumes a homogeneous occupation of all sites. This is the transition to the Mott-insulator phase where despite of the presence of the irregular lattice the ground state is dominated by the Fock state with uniform occupation numbers $n_{i}=N / I$. This transition between quasi Bose-glass and Mott-insulator phase happens independently of the lattice topology if the interaction strength $V$ exceeds the amplitude $\Delta$ of the lattice modulation.

The sequence of rearrangements in the quasi Bose-glass phase can be directly observed in experiment. The observable which is most sensitive to the changes in the spatial distribution of the atoms in the superlattice is the structure factor $S(k a)$ introduced in Sec. IVB. For a state with a homogeneous population of the lattice sites, e.g., in the Mott-insulator phase, $S(k a)$ shows peaks at integer values of $k a /(2 \pi)$ only. If mean-occupation numbers, i.e. the diagonal elements of the one-body density matrix, exhibit some periodic long-range structure then new peaks will appear at noninteger values of $k a /(2 \pi)$ from which information on the long-range order can be extracted.

The localized phase in a superlattice at small interaction strengths is the most pronounced realization of diagonal long-range order: For the superlattice discussed

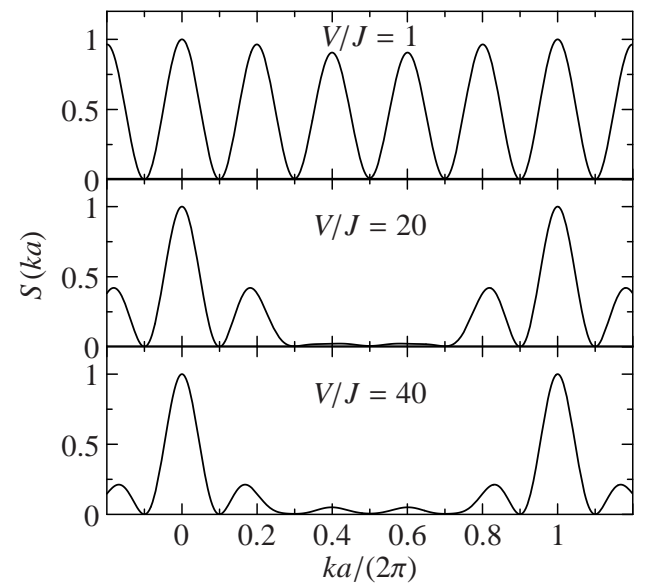

FIG. 13: Structure factor $S(k a)$ for different values of the interaction strength $V / J$ and fixed amplitude $\Delta / J=50$.

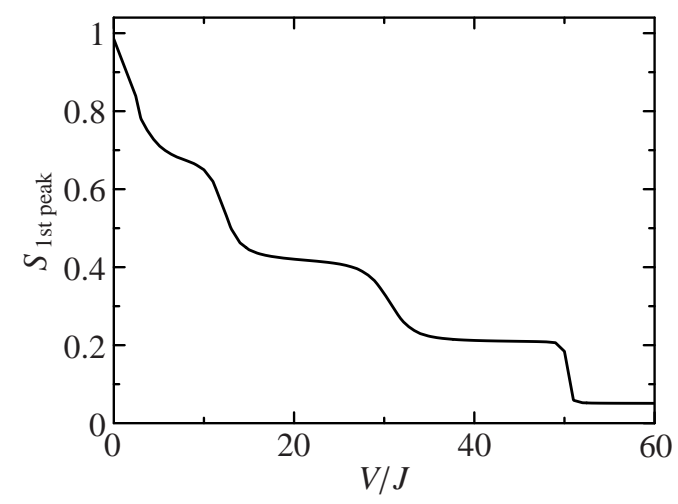

FIG. 14: Amplitude of the first peak of the structure factor $S(k a)$ located around $k a \approx 2 \pi / 5$ as function of the interaction strength $V / J$ for fixed $\Delta / J=50$.

here each 5th lattice site is occupied and all others are practically empty. The corresponding structure factor $S(k a)$ is shown in the upper panel of Fig. 13] Peaks of almost equal height emerge at $k a /(2 \pi)=\nu / 5$ ( $\nu$ integer $)$ indicating the perfect localization at a single site in each unit cell. With increasing interaction strength $V / J$ the particles are gradually redistributed to other lattice sites. This leads to a suppression of the peaks at non-integer values of $k a /(2 \pi)$ as the two lower panels Fig. 13 illustrate. Thus the amplitude of the peaks in $S(k a)$ provides detailed information on the complicated spatial structure within the quasi Bose-glass phase.

To highlight this point further, Fig. 14 shows the dependence of the amplitude of the first peak of $S(\mathrm{ka})$ located around $k a /(2 \pi) \approx 1 / 5$ as function of $V / J$ for fixed amplitude $\Delta / J$ of the lattice modulation. The parameters correspond to those used in Fig. 12 and cover the full range from the localized phase to the Mott-insulator. Obviously each of the rearrangements of the mean occupation numbers within the quasi Bose-glass phase leaves a distinct signature in the peak amplitude. The regions of stable configurations as well as the steplike rearrange- 

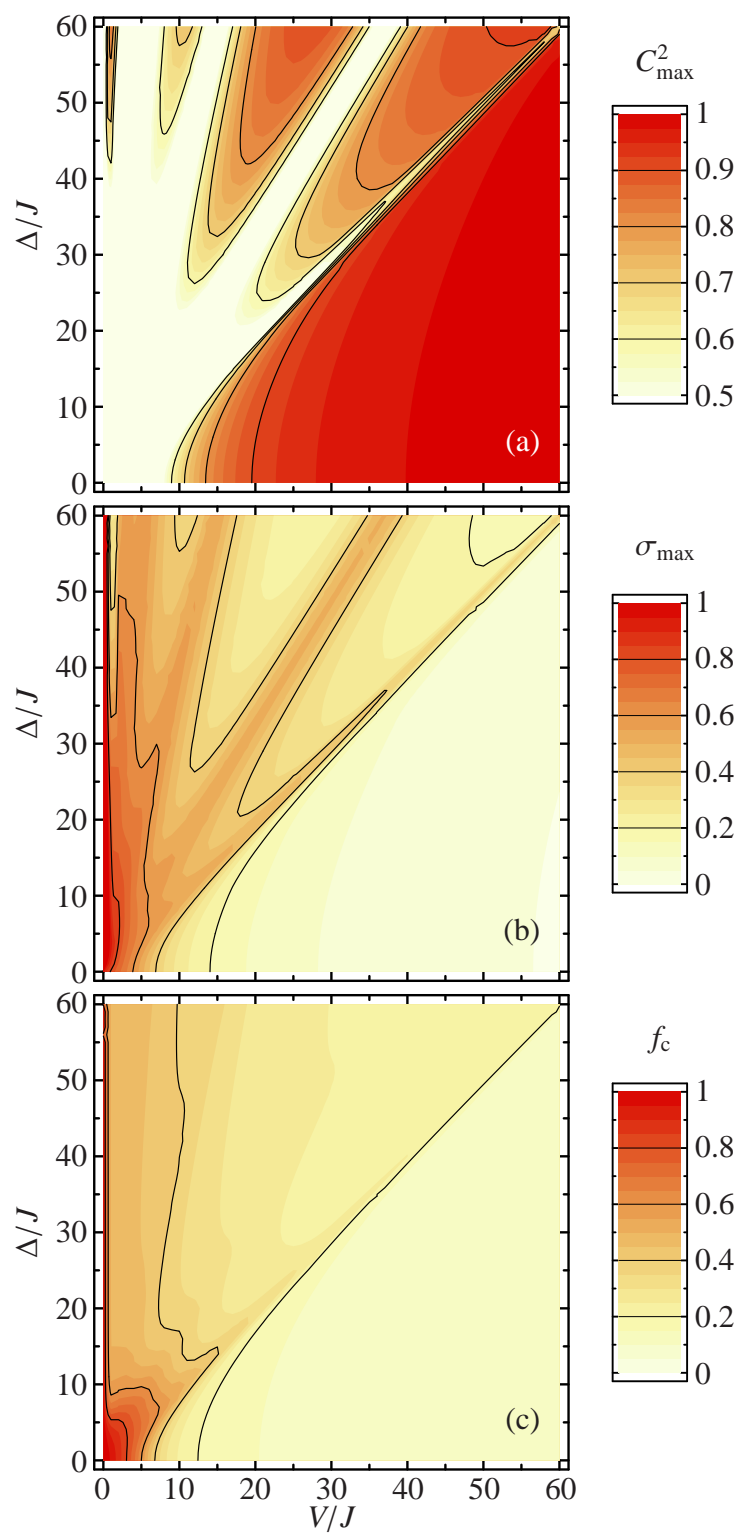

FIG. 15: Contour plots of the largest coefficient $C_{\max }$ (a), the maximum number fluctuations $\sigma_{\max }(\mathrm{b})$, and the condensate fraction $f_{\mathrm{c}}$ (c) in the $V-\Delta$ plane for the sinusoidal lattice with $I=N=10$.

ments that are visible in Fig. 12(a) directly show up in $S(k a)$. Thus the structure factor, which is experimentally accessible through Bragg scattering of light from the bosons in the lattice, provides a comprehensive insight into the spatial density structure and its complex dependency on the interaction strength and the modulation amplitude.

\section{Phase diagrams in the $V-\Delta$ plane}

We shall now illustrate the behavior of the superfluid fraction, condensate fraction, and energy gap using two- dimensional phase diagrams in the $V-\Delta$ plane. This representation exposes the complicated interplay between lattice modulation and two-body interaction which leads to the rich phase structure.

The gross phase structure is already revealed in the behavior of the largest coefficient $C_{\max }^{2}$ in the expansion (5) of the ground state which is depicted in Fig. 15(a). The dark shadings correspond to values of $C_{\max }^{2}$ close to 1, i.e. ground states which are almost pure Fock states. The prominent region of large $C_{\max }^{2}$ for values $V>\Delta$ corresponds to the Mott-insulator phase. The lobe-like structures at large modulation amplitudes $\Delta$ belong to the quasi Bose-glass phase. They correspond to the regions of stable configurations discussed in connection with Fig. 12] in which the mean occupation numbers are constant and approximately integral. The valleys of small $C_{\max }^{2}$ which separate the lobes are connected to the rearrangements between different stable configurations. As mentioned earlier the number and position of these lobes depends on the topology of the lattice potential.

In close connection with the behavior of $C_{\max }^{2}$ are the maximum number fluctuations $\sigma_{\max }=\max \left(\sigma_{i}\right)$ depicted in Fig. 15(b). Values of $C_{\max }^{2}$ close to 1 imply that the number fluctuations at all lattice sites are small. Thus the Mott-insulator phase and the lobes of the quasi Boseglass phase appear as regions of small number fluctuations (light shadings). In contrast, the rearrangement valleys in the quasi Bose-glass phase, which are associated with small $C_{\max }^{2}$, show increased number fluctuations. Number fluctuations of the order 1 emerge only within a narrow band at small values of $V / J$. For large values of $\Delta / J$ this corresponds to the localized phase discussed in Sec. $\mathrm{VB}$

The behavior of the condensate fraction $f_{\mathrm{c}}$ is shown in Fig. 15(c). In line with variations of the maximum number fluctuations, $f_{\mathrm{c}}$ assumes very small values in the Mott-insulator phase. Within the quasi Bose-glass it decreases monotonically with increasing $V / J$, i.e., it does not show the lobe structure as the number fluctuations. Values of the condensate fraction above 0.6 result only in a small area at small $V / J$ and $\Delta / J$ and a very narrow stripe at $V / J \lesssim 2$. Thus only the localized phase and the expected superfluid phase exhibit sizeable condensate fractions.

So far, these quantities are determined by the properties of the ground state of Bose gas. Following the discussion in Sec. [IIB they therefore do not fully determine the superfluid properties of the system. Figure 16 shows the contour plots for two quantities which probe the excitation spectrum, these are the superfluid fraction and the energy gap. The plot for superfluid fraction $f_{\mathrm{s}}$ in Fig. 16/a) pinpoints the boundaries of the superfluid phase. Large values of the superfluid fraction (dark shadings) result only in a restricted parameter region at small values of $V$ and $\Delta$. Everywhere else - that is, in the Mott-insulator, the quasi Bose-glass, and the localized phase - the system is a perfect insulator. In comparing the boundaries of the superfluid phase defined in Fig. 


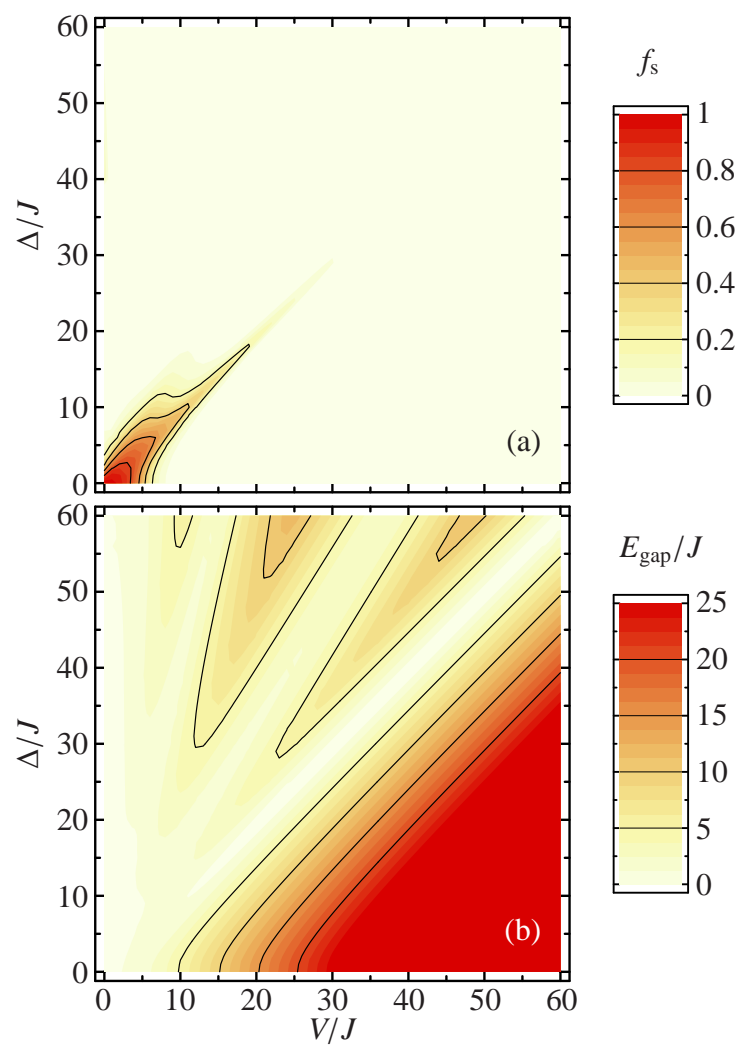

FIG. 16: Contour plots of the superfluid fraction $f_{\mathrm{s}}$ (a) and the energy gap $E_{\text {gap }}(\mathrm{b})$ for the sinusoidal lattice with $I=$ $N=10$.

16(a) with the structure of the other observables, like number fluctuations and condensate fraction in Fig. [15] it becomes evident that none of the ground state quantities can identify the superfluid region. Not only that the magnitude of the superfluid fraction cannot be determined, the whole structure of the plots is different.

This also applies to the energy gap $E_{\text {gap }}$ plotted in Fig. 16(b). Although the opening of a gap between the ground state and the first excited state is an indicator for the superfluid to Mott-insulator transition in the uniform lattice, there is no such correspondence for the irregular lattice. It is only the Mott-insulator phase that exhibits a large energy gap. The localized and the quasi Bose-glass phase, which are also insulating, have small energy gaps just like the superfluid phase.

The energy gap of the quasi Bose-glass phase deserves closer inspection. Figure [16(b) reveals that there is a small but finite energy gap within the stable lobes of the quasi Bose-glass phase. The size of the gaps, as well as the position and number of the lobes, depend on the differences between the various on-site energies. If the size of the unit cells is increased or if one goes to large random lattices then the differences between the $\epsilon_{i}$ become smaller, the number of lobes increases, their size decreases, and the energy gap vanishes. Thus there is a continuous transition from the quasi Bose-glass to the strict Bose-glass phase with vanishing $E_{\text {gap }}$.
Another interesting observation results from the comparison of the superfluid fraction in Fig. 16) a) with the condensate fraction in Fig. 15(c). In an irregular lattices with weak interactions there is a region where the condensate fraction is larger than the superfluid fraction. An extreme case is the localized phase at $V / J \lesssim 2$, where $f_{\mathrm{s}} \approx 0$ and $f_{\mathrm{c}} \approx 1$. This means that the localized bosons in the different unit cells are still phase coherent but the superfluid flow is inhibited by the lattice topology. A similar phenomenon was recently observed in a Monte Carlo study of a continuous Bose gas with random impurities [40] which act like the irregular lattice potential.

So far we have only considered the phase diagrams for a filling factor $N / I=1$. The qualitative structure of the phase diagram does not change if we go to larger integer filling factors. Quantitatively the superfluid region expands towards larger values of the interaction strength $V / J$ and the modulation amplitude $\Delta / J$ if the filling factor is increased. For a filling factor $N / I=4$, for example, the contour corresponding to a superfluid fraction $f_{\mathrm{s}}=0.6$ extends up to $V / J \approx 15$ and $\Delta / J \approx 40$. The insulating phases are shifted accordingly.

\section{E. Non-commensurate filling factors}

It is well-known that the Mott-insulator phase in a uniform lattice exists only for commensurate filling factors. If one adds a few particles on top of a commensurate configuration then these particles can be moved to any lattice site without changing the energy - even in the presence of strong repulsive interactions. In this way the additional particles gives rise to a finite superfluid fraction in the limit of large $V / J$.

This intuitive picture is confirmed by the exact calculations for the sinusoidal superlattice with $I=10$ sites and $N=12$ particles. Contour plots for the superfluid fraction and the energy gap in the $V-\Delta$ plane are presented in Fig. 17. For the uniform lattice $(\Delta / J=0)$ the superfluid fraction shown in panel (a) remains finite even at large $V / J$.

If one starts to increase the amplitude $\Delta$ of the sinusoidal modulation then the superfluid fraction decreases and eventually vanishes above $\Delta / J \gtrsim 5$. In this regime the growing lattice irregularity causes a pinning of the additional particles to the lattice sites with minimal onsite energies $\epsilon_{i}$. The ground state has an increased occupation number at the deepest lattice well of each unit cell.

The behavior of the energy gap shown in Fig. 17(b) confirms this picture. For strong repulsive interactions the energy gap vanishes at small amplitudes $\Delta / J$ in stark contrast to the commensurate system. With increasing amplitude of the sinusoidal modulation the energy gap starts to grow linearly with $\Delta / J$. In this simple case $E_{\text {gap }}$ corresponds to the energy required to remove the additional particle from the lattice site it is affixed to. The slope of the increase is proportional to the energy 


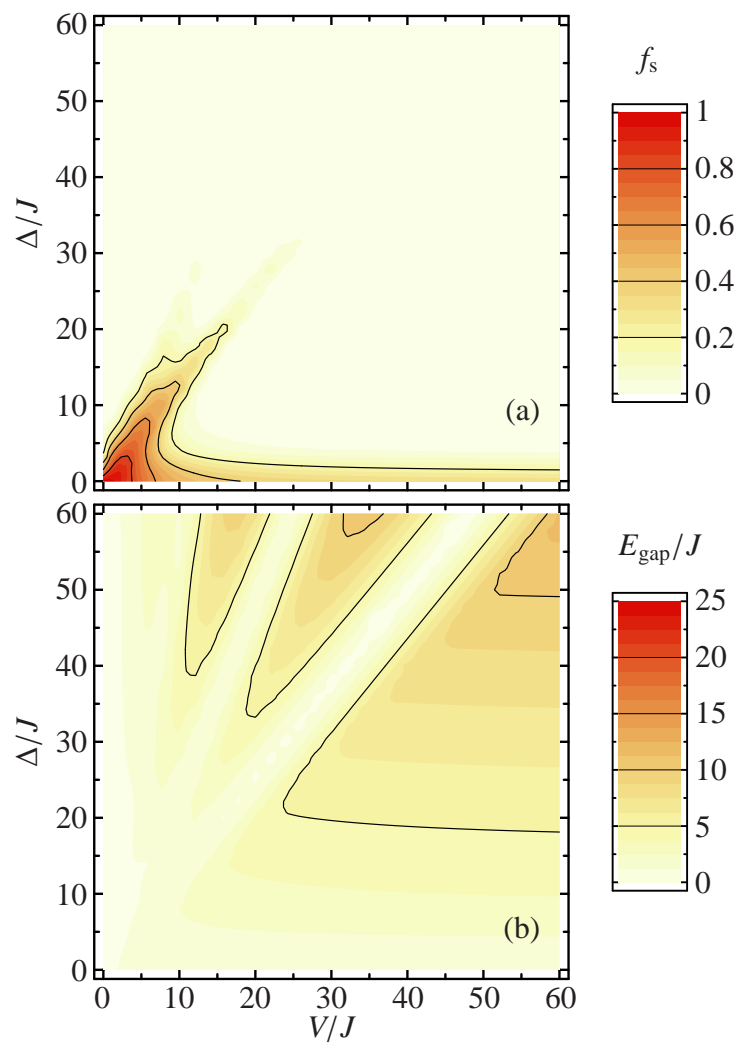

FIG. 17: Contour plots of the superfluid fraction $f_{\mathrm{s}}(\mathrm{a})$ and the energy gap $E_{\text {gap }}$ (b) in the $V-\Delta$ plane for the sinusoidal lattice with $I=10$ and non-commensurable particle number $N=12$.

difference between the two lowest $\epsilon_{i}$. This behavior is equivalent to the structure of the lobes of the quasi Boseglass phase.

Thus, in general, the change from a commensurate to a non-commensurate filling factor causes a vanishing of the Mott-insulator phase. It is replaced by a superfluid phase at small $\Delta / J$, where the additional particles constitute a superfluid on top of an Mott-insulating layer. At larger $\Delta / J$ the additional particles are pinned to the deepest lattice wells, superfluidity ceases, and an additional lobe of the quasi Bose-glass phase emerges. All other regions of the phase diagram are qualitatively unaffected by the change from a commensurate to a non-commensurate filling factor.

\section{CONCLUSIONS}

We have shown that the superfluid fraction - the natural order parameter for superfluid to insulator phase transitions - is determined by the response of the system to an external perturbation, i.e., a twist in the boundary conditions, or equivalently an additional phase factor, in the tunneling term of the Bose-Hubbard Hamiltonian. This means that the superfluid fraction necessarily depends on the full excitation spectrum of the lattice sys- tem. The formal manifestation of this fact is given in Eq. (24), where the superfluid fraction is expressed as a sum of a first order term depending only of the ground state and a second order term which involves all excited states. The exact numerical solution of the Bose-Hubbard model for uniform one-dimensional lattices reveals that the second order term is crucial for the vanishing of the superfluid fraction in the Mott-insulator phase. The first order contribution gives only an upper bound for the superfluid fraction and can have a rather large value in the insulating phase.

This allows important conclusions for the prospects of measuring the superfluid fraction and the details of superfluid to insulator phase transitions. Present experimental observables are sensitive only to the ground state and therefore cannot probe the physics that governs the superfluid properties. The matter-wave interference pattern, for example, provides direct information on the quasimomentum distribution of the ground state but it does not measure the superfluid properties. In the case of the superfluid to Mott-insulator transition our numerical results reveal that the superfluid fraction vanishes much earlier than the interference fringes, i.e., an interference pattern is still visible in the Mott-insulator phase. This demonstrates that one has to clearly distinguish between superfluidity and coherence properties.

In the second part of the paper we have employed these tools to explore the zero-temperature phase diagram of Bose gases in non-uniform lattice potentials. We have demonstrated that even a simple superlattice potential which results from the superposition of two standingwave lattices with different wavelengths gives rise to a very rich phase diagram. As function of the interaction strengths $V / J$ and the amplitude $\Delta / J$ of the spatial variation of the on-site energies two additional insulating phases can be identified: a localized phase and a quasi Bose-glass phase. All insulating phases can be clearly distinguished through their characteristic signatures in matter-wave interference experiments or through the structure factor determined by Bragg diffraction of light.

Our results further support the view that Bose gases in optical lattices are a versatile tool for studying quantum mechanical many-body phenomena in strongly correlated systems. They allow us to address fundamental questions such as the connection between superfluidity and BoseEinstein condensation. Moreover, they enable controlled studies of disorder-induced phenomena which result from a subtle competition between kinetic energy, two-body interaction, and lattice topology. To this end two-color lattices with relatively small unit cells seem a promising starting point because they facilitate a clear experimental distinction between the various phases. 


\section{Acknowledgments}

This work was supported by the DFG, the UK EP$\mathrm{SRC}$, and the EU via the "Cold Quantum Gases" net- work. Keith Burnett thanks the Royal Society and Wolfson Foundation for support. We acknowledge useful discussions with S.A. Gardiner, J.A. Dunningham, T. Köhler, T. Gasenzer, and K. Braun-Munzinger.
[1] M. Greiner, O. Mandel, T. Esslinger, T. W. Hänsch, and I. Bloch, Nature 415, 39 (2002).

[2] M. Greiner, O. Mandel, T. W. Hänsch, and I. Bloch, Nature 419, 51 (2002).

[3] S. Sachdev, Quantum Phase Transitions (Cambridge University Press, Cambridge, 1999).

[4] D. Jaksch, C. Bruder, J. I. Cirac, C. W. Gardiner, and P. Zoller, Phys. Rev. Lett. 81, 3108 (1998).

[5] R. Roth and K. Burnett, Phys. Rev. A 67, 031602(R) (2003).

[6] R. T. Scalettar, G. G. Batrouni, and G. T. Zimanyi, Phys. Rev. Lett. 66, 3144 (1991).

[7] W. Krauth, N. Trivedi, and D. Ceperley, Phys. Rev. Lett. 67, 2307 (1991).

[8] R. Roth and K. Burnett, to be published in J. Opt. B (2003), cond-mat/0205412.

[9] C. Orzel, A. K. Tuchman, M. L. Fenselau, M. Yasuda, and M. A. Kasevich, Science 291, 2386 (2001).

[10] M. P. A. Fisher, P. B. Weichman, G. Grinstein, and D. S. Fisher, Phys. Rev. B 40, 546 (1989).

[11] G. G. Batrouni, R. T. Scalettar, and G. T. Zimanyi, Phys. Rev. Lett. 65, 1765 (1990).

[12] D. van Oosten, P. van der Straten, and H. T. C. Stoof, Phys. Rev. A 63, 053601 (2001).

[13] B. Damski, J. Zakrzewski, L. Santos, P. Zoller, and M. Lewenstein (2003), cond-mat/0303065.

[14] G. G. Batrouni and R. T. Scalettar, Phys. Rev. B 46, 9051 (1992).

[15] V. A. Kashurnikov, N. V. Prokof'ev, and B. V. Svistunov, Phys. Rev. A 66, 031601(R) (2002).

[16] G. G. Batrouni, V. Rousseau, R. T. Scalettar, M. Rigol, A. Muramatsu, P. J. H. Denteneer, and M. Troyer (2002), cond-mat/0203082.

[17] W. Krauth, M. Caffarel, and J.-P. Bouchaud, Phys. Rev. B. 45, 3137 (1992).

[18] A.-M. Rey, K. Burnett, R. Roth, M. Edwards, C. J. Williams, and C. W. Clark, J. Phys. B 36, 825 (2003).

[19] J. K. Freericks and H. Monien, Phys. Rev. B 53, 2691 (1996).
[20] S. Sachdev, K. Sengupta, and S. M. Girvin, Phys. Rev. B 66, 075128 (2002).

[21] K. Braun-Munzinger, J. A. Dunningham, and K. Burnett (2002), cond-mat/0211701.

[22] F. Dalfovo, S. Giorgini, L. P. Pitaevskii, and S. Stringari, Rev. Mod. Phys. 71, 463 (1999).

[23] O. Penrose and L. Onsager, Phys. Rev. 104, 576 (1956).

[24] C. N. Yang, Rev. Mod. Phys. 34, 694 (1962).

[25] W. Jones and N. H. March, Theoretical Solid State Physics, Volume 1: Perfect Lattices in Equilibrium (Dover Publications, New York, 1985).

[26] E. L. Pollock and D. M. Ceperley, Phys. Rev. B 36, 8343 (1987).

[27] E. H. Lieb, R. Seiringer, and J. Yngvason (2002), condmat/0205570.

[28] A. J. Leggett, Rev. Mod. Phys. 71, S318 (1999).

[29] M. E. Fisher, M. N. Barber, and D. Jasnow, Phys. Rev. A 8, 1111 (1973).

[30] D. Poilblanc, Phys. Rev. B 44, 9562 (1991).

[31] B. S. Shastry and B. Sutherland, Phys. Rev. Lett. 65, 243 (1990).

[32] W. Krauth, Phys. Rev. B 44, 9772 (1991).

[33] K. G. Singh and D. S. Rokhsar, Phys. Rev. B 49, 9013 (1994).

[34] R. M. Fye, M. J. Martins, D. J. Scalapino, J. Wagner, and W. Hanke, Phys. Rev. B 44, 6909 (1991).

[35] A. Paramekanti, N. Trivedi, and M. Randeria, Phys. Rev. B 57, 11639 (1998).

[36] G. Birkl, M. Gatzke, I. H. Deutsch, S. L. Rolston, and W. D. Phillips, Phys. Rev. Lett. 75, 2823 (1995).

[37] M. Weidemüller, A. Hemmerich, A. Görlitz, T. Esslinger, and T. W. Hänsch, Phys. Rev. Lett. 75, 4583 (1995).

[38] A. van Otterlo, K.-H. Wagenblast, R. Baltin, C. Bruder, R. Fazio, and G. Schön, Phys. Rev. B 52, 16176 (1995).

[39] L. Guidoni, C. Triché, P. Verkerk, and G. Grynberg, Phys. Rev. Lett. 79, 3363 (1997).

[40] G. E. Astrakharchik, J. Boronat, J. Casulleras, and S. Giorgini, Phys. Rev. A 66, 023603 (2002). 